\title{
In Vivo Methods for the Assessment of Topical Drug Bioavailability
}

\author{
Christophe Herkenne, ${ }^{1,2}$ Ingo Alberti, ${ }^{1,3}$ Aarti Naik, ${ }^{1}$ Yogeshvar N. Kalia, ${ }^{1}$ François-Xavier Mathy,, 4 \\ Véronique Préat, ${ }^{4}$ and Richard H. Guy ${ }^{1,6,7}$
}

Received June 5, 2007; accepted July 24, 2007; published online November 6, 2007

\begin{abstract}
This paper reviews some current methods for the in vivo assessment of local cutaneous bioavailability in humans after topical drug application. After an introduction discussing the importance of local drug bioavailability assessment and the limitations of model-based predictions, the focus turns to the relevance of experimental studies. The available techniques are then reviewed in detail, with particular emphasis on the tape stripping and microdialysis methodologies. Other less developed techniques, including the skin biopsy, suction blister, follicle removal and confocal Raman spectroscopy techniques are also described.
\end{abstract}

KEY WORDS: cutaneous bioavailability; cutaneous drug concentration; dermatopharmacokinetics; microdialysis; tape stripping.

\section{INTRODUCTION}

Skin is the largest organ of the human body and is composed of three readily distinguishable layers: (a) the stratum corneum (SC), which is the outermost part of the epidermis, (b) the living epidermis, and (c) the dermis, penetrated by a highly complex network of capillaries involved in the removal of drugs from the skin into the systemic circulation. In addition, several pilosebaceous and sweat glands are dispersed throughout the skin, in various numbers and size, depending on body site. The SC is wellrecognized as a heterogeneous two-compartment system of flattened keratinized cells embedded in a multilamellar lipid matrix mainly composed of neutral lipids and ceramides (1-4). It has a thickness of about $10 \mu \mathrm{m}(5-7)$ and is composed of about 20 cell layers (5). Its main homeostatic functions are restriction of excessive water loss to the external environment, moisture retention, cell cohesion and desquamation. In addition, SC is considered to be the ratecontrolling membrane for transport of xenobiotics across the skin (although, for very lipophilic molecules, partitioning into

\footnotetext{
${ }^{1}$ School of Pharmaceutical Sciences, University of Geneva, 30 quai E. Ansermet, CH-1211, Geneva 4, Switzerland.

${ }^{2}$ Debio Recherche Pharmaceutique S.A., Route du Levant 146, CH1920, Martigny, Switzerland.

${ }^{3}$ Antares Pharma AG, Gewerbestrasse 18, 4123, Allschwil, Switzerland.

${ }^{4}$ Université catholique de Louvain, Unité de pharmacie galénique, industrielle et officinale, Avenue E. Mounier 73, 1200, Brussels, Belgium.

${ }^{5}$ UCB SA, Chemin du Foriest 1, 1420 Braine-l'Alleud, Belgium.

${ }^{6}$ Department of Pharmacy \& Pharmacology, University of Bath, Claverton Down, Bath, BA2 7AY, Allschwil, UK.

${ }^{7}$ To whom correspondence should be addressed.(e-mail: r.h.guy@ bath.ac.uk)
}

the more hydrophilic viable tissue may constitute the greater resistance $(2,8))$. Therefore, topical skin bioavailability assessment of xenobiotics is essential for both the (trans)dermal delivery of pharmacological active drugs and from a toxicological point of view (9).

For systemically delivered oral products, bioavailability is typically defined as the rate and extent at which a drug reaches the general circulation from an administered dosage form. Dermatological drug products include a broad array of preparations which are designed to exert a local effect in diseased skin following topical application on the skin surface. The objective is to maximize drug concentration at the site of action within the skin with, ideally, a minimal systemic uptake $(10,11)$. Thus, systemic availability may not properly reflect local cutaneous bioavailability (as it does for transdermal products, on the other hand, which are designed to deliver drug into the systemic circulation). Moreover, topical doses tend to be so small (typically 2-5 $\mathrm{mg}$ of product $/ \mathrm{cm}^{2}$ ) that serum and/or urine concentrations are often undetectable using conventional assay techniques (12). A further complicating factor is the lack of knowledge of the drug concentration needed at the skin target site (with the exception of antifungal agents whose target site is the SC surface). Topical bioavailability has been more rigorously defined as the temporal pattern of free drug concentration at the target site [the so-called $\mathrm{C}^{*}$ concept (10)], but the value of this approach remains largely theoretical due to the difficulty of quantifying drug within the skin.

What options are available, then, for assessment of topical bioavailability and bioequivalence? Although a drug's permeability coefficient through human skin can be estimated, for example, from the molecular weight and octanol-water partition coefficient (13), this information is not sufficient to estimate topical bioavailability. The algorithms available are 
able to approximate a value for the maximum drug flux across the skin, but cannot quantify the potential impact of particular vehicles (or vehicle constituents) on skin barrier function (14). Equally, these calculations assume that the thermodynamic activity of the drug remains constant during the application period, a situation which does not pertain, of course, when one or more formulation component is volatile (15) or the dose administered is very small (i.e., a finite dose situation).

It follows that an experimental approach is necessary, and the objective of this review is to evaluate different techniques currently under consideration. We begin with a discussion of the different procedures which are accepted by the regulatory authorities at this time, and which contribute to the approval of innovative, and/or generic, topical drug products.

\section{CURRENT OPTIONS FOR TOPICAL BIOAVAILABILITY/BIOEQUIVALENCE ASSESSMENT}

As mentioned above, for topical dermatological products, pharmacokinetic measurements in blood, plasma or urine are usually not feasible because of the very low concentrations achieved in these typical sampling compartments. There is also debate as to whether measurable levels in the blood (when detectable), for example, are relevant to local, topical drug bioavailability. On the other hand, the cumulative amount of drug reaching the systemic circulation is pertinent with respect to potential toxicity and may indicate the need to limit topical application to avoid undesirable side-effects.

For certain topical drugs, specifically the corticosteroids, pharmacodynamic measurements represent an accepted approach with which to establish bioequivalence between different formulations (and are used, as well, in the development of new chemical entities in the same therapeutic class). These drugs produce skin blanching at the site of application and this response has been correlated with clinical efficacy (16). The original Stoughton-McKenzie vasoconstriction assay (17) employed visual evaluation of the degree of blanching by trained observers. As this approach is rather subjective (18), it is now considered sensible to use a chromameter to quantify the blanching response (19-22). This method has been reviewed in detail elsewhere (20).

While the advantages of the vasoconstriction assay for topical steroids are clear, the approach has the obvious limitation that it cannot be applied to all drugs. Indeed, the literature contains very few other examples in which pharmacologic response has been used to assess topical drug availability. Evaluation of vasodilatation provoked by nicotinic acid esters, and its inhibition by anti-inflammatory drugs, has been performed using laser Doppler velocimetry and chromametry (23-27). The reduction in erythema induced by topical ibuprofen has been correlated with drug levels in the epidermis (27). Equally, topical retinoids have been shown to provoke increases in transepidemal water loss in a time- and dose-dependent fashion (28-30), although the generality of this effect has not been established (29).

It follows that, at the present time, topical drug bioavailability and bioequivalence must be assessed via clinical studies, which are typically costly and time- consuming (31); regulatory agencies have therefore been seeking alternative methodologies, especially for bioequivalence studies to ease the burden on those attempting to commercialize useful products at competitive prices. While much debate has centered on the potential of different in vitro techniques, in particular the use of Franz diffusion cell studies to compare different formulations, these methods have not been accepted as useful contributors to the overall determination of bioavailability and bioequivalence. Currently, the relevance, reliability and reproducibility of the in vitro approach have not been demonstrated to the level necessary for the regulatory bodies; major concerns centre around the source and supply of human skin tissue, the 'quality-control' of the experiments, in general, and the absence of an effective clearance mechanism for drugs (especially lipophilic species) from the membrane. Only for demonstrating the benign effect of minor formulation changes are in vitro experiments, using model, polymer membranes, authorized by, for example, the US Food and Drug Administration.

Nevertheless, there remains a real need to develop valid, effective and economical approaches with which to measure topical drug bioavailability, not least for the evaluation of bioequivalence when generic products are submitted for marketing approval. The remainder of this review examines, primarily, two methodologies currently under close examination for the measurement of topical bioavailability and bioequivalence: namely, the tape-stripping and microdialysis techniques.

\section{TAPE STRIPPING}

Tape stripping involves sequentially removing microscopic layers (typically $0.5-1 \mu \mathrm{m}$ ) of stratum corneum. It is usually performed by placing an adhesive tape-strip onto the skin surface, followed by gentle pressure to ensure a good contact, and subsequently removal by a sharp upward movement. The procedure is relatively painless and noninvasive, given that only dead cells (corneocytes) embedded in their lipid matrix are removed. Even if skin stripping remains a form of environmental insult, a homeostatic repair response in the epidermis is rapidly elicited (32) which results in rapid restoration of barrier function (33).

Tape stripping is used in various fields of cutaneous biology: for example, to evaluate the barrier function (34), to investigate dermatopathologies such as inflammatory or neoplastic disorders or xerotic conditions (35), to monitor gene expression $(36,37)$, to investigate $\mathrm{pH}$ profiles $(38)$, to evaluate animal skin as a surrogate for human skin (39), to induce Toll-like receptor 9 expression (40) and so on (41). In dermatopharmacology, tape stripping is used to assess cutaneous drug or excipient levels in the skin after topical dermatological treatment, either in the removed tape-strips, or directly in the tape-stripped skin. It follows that tape stripping is a particularly useful technique to assess the local bioavailability of drugs whose target site is the SC itself (such as antifungal agents (43-46), UVA/UVB filters (47-51) or antiseptics (52).

The tape stripping technique may also offer a real alternative for local bioavailability and/or bioequivalence assessment of topically applied dermatological agents whose 
target is the underlying viable tissues. As the SC is usually the principal resistance to the penetration of topically applied compounds (4), it has been argued that drug levels in the barrier should be correlated with those attained in the underlying, viable components of the skin, i.e., the sites at which many dermatological diseases are manifest.

This hypothesis was tested, but only partially validated, by Rougier et al. (53-56). In both animal models and human volunteers, for a range of compounds (mostly low molecular weight, low lipophilicity) delivered from several different formulations, the amount of active in the SC after a $30-\mathrm{min}$ application, and subsequent removal of any remaining vehicle, was determined by tape-stripping. At a contra-lateral position, the same 30-min application and cleaning procedure was adopted but, rather than tape-stripping the SC, the penetration of chemical taken up into the skin was quantified by its urinary excretion over the next 4 days. Good correlation between the two measurements was achieved (see Fig. 1) suggesting the value of the tape-stripping approach for a simple and rapid determination of potential systemic exposure. However, the experiment only solves part of the bioavailability challenge: it provides a measurement of the extent of absorption but gives no information on rate. As such, in its originally developed form, the so-called Rougier technique is not suitable for regulatory use.

An alternative strategy to test the hypothesis is to demonstrate correlation between drug levels measured in the SC by tape-stripping and a pharmacodynamic effect in the skin. This has been accomplished in some necessarily limited studies using (a) hydrocortisone (57) and betamethasone diproprionate $(16,20)$, the pharmacological activity of which was assessed by skin blanching, (b) miconazole and ketoconazole, the efficacy of which against $C$. Albicans in vitro was

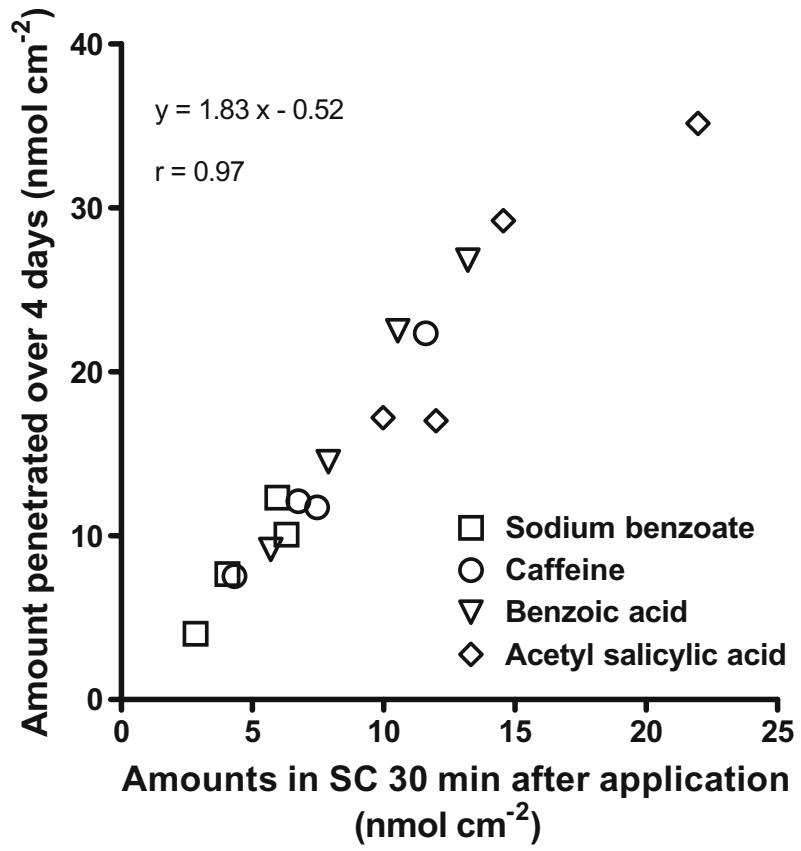

Fig. 1. Correlation between the amount of different chemicals absorbed across skin following a 30-min application and the quantity recovered in SC tape-strips after an identical, but independent, administration procedure. Redrawn from (52). determined (42), and (c) iododeoxyuridine, for which clinical efficacy in reducing lesion severity was evaluated (58). Ultimately, of course, these types of validation studies are essential if a tape-stripping approach is to be adopted for routine bioavailability and bioequivalence assessment. For the moment, however, the field is (appropriately) examining how to optimize the tape-stripping procedure so as to extract the maximum information from the experiment in the most efficient manner possible. These advances are now discussed in detail.

\section{Method Development and Optimization}

Sequential tape-stripping of the stratum corneum allows horizontal fractions of the membrane to be obtained. The tape-strips must then be extracted to recover and quantify the absorbed drug. Local bioavailability may be assessed either from the combined tape strips, or from the individual tape-strips. The method of quantification depends, of course, on the nature of the drug and the amount present on the tape-strips. Various approaches have been used ranging from UV-VIS spectrophotometry (59) to the more ubiquitous HPLC (with UV, fluorescence, or even mass spectrophotometric detection), gas chromatography (60-62), scintillation counting $(42,57,63)$ and infrared spectroscopy (64-67). The key criteria are that the extraction process does not degrade the drug, that it is efficient and reproducible, and that it is free from interference from components of the SC and/or the tape adhesive. Quantification of the drug in the combined tape-strips enables the total amount in the stratum corneum to be determined.

After much discussion (31,68-71), a draft guidance was published by the US Food and Drug Administration (FDA) in 1998 (21) in which the general procedures for conducting a bioavailability/bioequivalence study were described. Briefly, the guidance proposed the following steps:

1. After application of a drug product for a given application time, excess formulation was to be removed by an appropriate (but not specified) procedure.

2. Two sequential tape-strips of the SC were to be taken and discarded, the guidance arguing that material located on these samples had not been, and would not be, absorbed.

3. Subsequently, ten strips from the application site were to be made, combined and extracted with a suitable solvent.

4. The entire process was to be performed at multiple sites following different times of application (absorption phase, Fig. 2a) and different periods between the longest application time and the time at which stripping was performed (elimination phase, Fig. 2b).

5. The total amount of drug in the SC was then to be displayed as a function of time as a so-called dermatopharmacokinetic (DPK) profile, characterized by a maximum amount, the time at which this maximum was achieved, and an 'area-under-thecurve' (Fig. 3).

6. Simultaneous comparison of two formulations of the same drug would allow an assessment of local bioequivalence using the representative DPK parameters. 


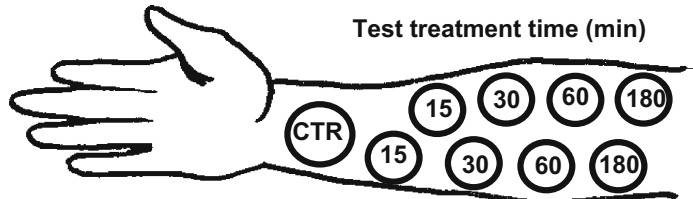

Reference treatment time $(\mathrm{min})$

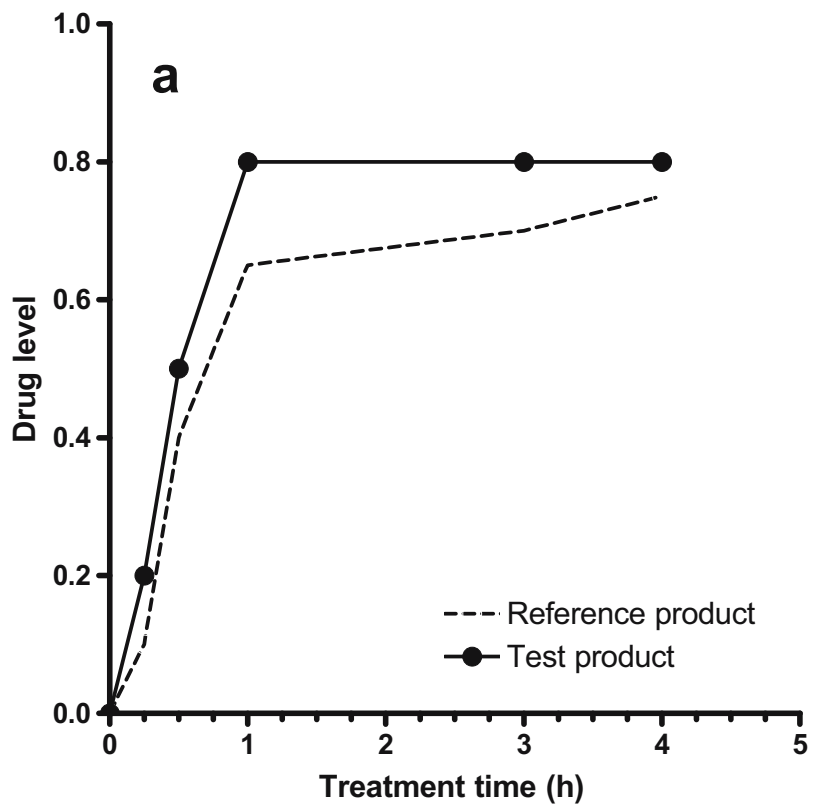

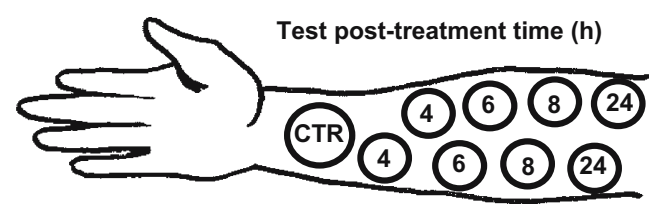

Reference post-treatment time (h)

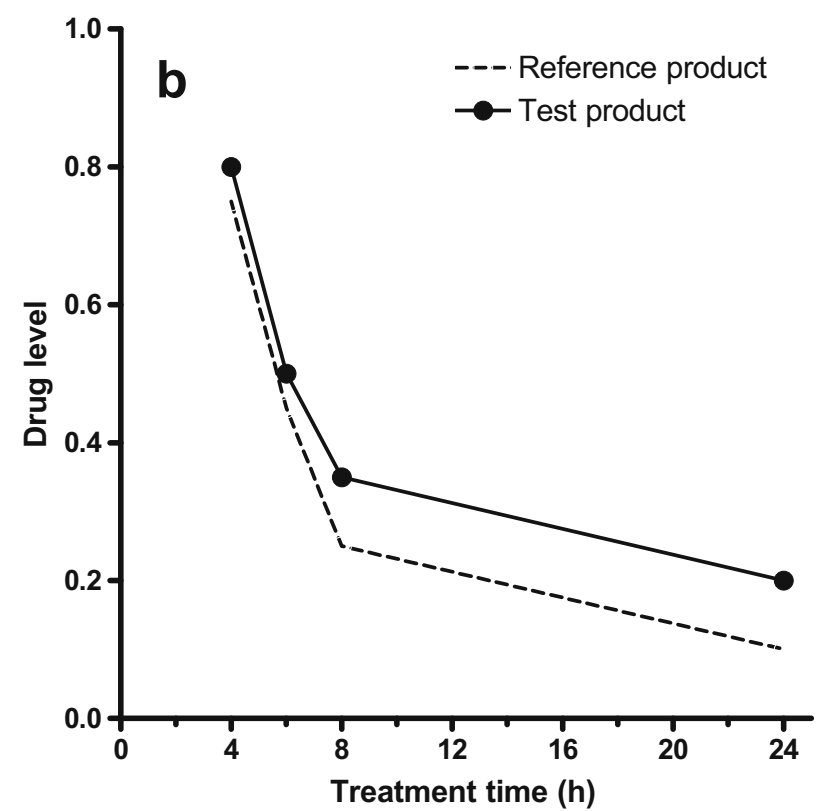

Fig. 2. Schematic representation of a dermatopharmacokinetic (DPK) study to assess topical bioequivalence between test and reference formulations containing the same drug. a In the uptake, or absorption, phase, the SC is tape-stripped immediately after each treatment time, and the drug level in the barrier is determined. b In the clearance phase, after the maximum treatment time, the SC is subsequently stripped after progressively longer periods post-removal of the formulations. $C T R=$ control, drug-free formulation.

Although useful as a 'jumping-off' point, the guidance was open to a number of criticisms, and exposed some clear flaws. In brief, while many of these problems have now been recognized, but not fully resolved, the document has therefore been withdrawn and, at the time of writing, the possibility of a refined version being developed is under examination. Among the weaknesses of the original guidance, one may cite, in particular:

(a) The timing of the tape-stripping procedures during the absorption and elimination phases is not clearly delineated and (apart from trial-and-error) no rational approach to their determination is offered.

(b) The number of tape-stripping procedures is considerable, rendering a DPK evaluation onerous in terms of the work involved (despite the fact that 'pooling' tapes for analysis does partly reduce this burden).

(c) The unvalidated discarding of information from the first two tape-strips has never been subjected to rigorous examination; indeed, it has been argued that these data are relevant to a complete bioavailability assessment (46).

(d) Ten tape-strips do not remove the same amount of SC from all subjects (or even, potentially, within a subject, especially if one of the formulations being compared contains an excipient which weakens SC cohesivity). As a result, drug will be measured in

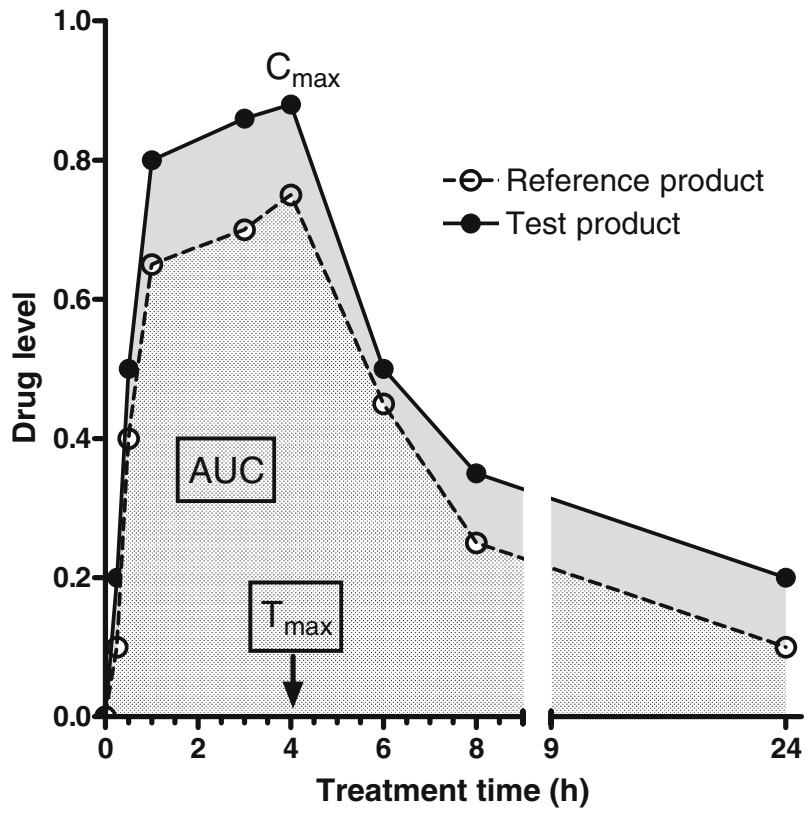

Fig. 3. DPK profiles of reference and test formulations containing the same drug. The parameters to be compared are the maximum drug level in the SC $\left(C_{\max }\right)$, the time at which this maximum level is reached $\left(T_{\max }\right)$ and the area under the SC quantity versus time profile $(A U C)$. 
different 'volumes' of the matrix, rendering comparisons between different treatments/subjects essentially meaningless.

Nevertheless, there have been some reports of success using the DPK procedure as outlined in the FDA guidance (or at least part of it); one particularly good example showed a correlation between both the area-under-the-curve and maximum concentration parameters and the clinical efficacy of tretinoin gels (Fig. 4 (72)). Less encouraging, and ultimately responsible for the DPK guidance's withdrawal, was an inter-laboratory comparative study (again using tretinoin) that resulted in diametrically opposite conclusions about the bioequivalence of different formulations (73-75).

The outcome of this initial work has been a serious reevaluation of the overall worthiness of the approach and an ongoing examination of how it may be improved. The need to quantify (or, at least, to have some idea of the amount of) the SC removed is now clear, not only for the reasons already given, but because it is obvious that different tapes and different experimentalists will inevitably strip the SC in a variable fashion, making inter- and intra-laboratory comparisons impossible. Even when the type of tape is standardized, and an attempt is made to exert an equal pressure on each tape applied to the skin (76), the SC amount removed still depends on, for example, the speed with which the tape is subsequently removed from the subject and exactly where on the forearm the experiment is performed. As standardization appears very difficult to achieve, therefore, it is clearly more sensible to identify a method by which the SC removed can be quantified, thereby allowing the uptake of drug into this tissue to be normalized (and expressed, for example, as an amount per unit volume, or per unit weight, of SC). For the

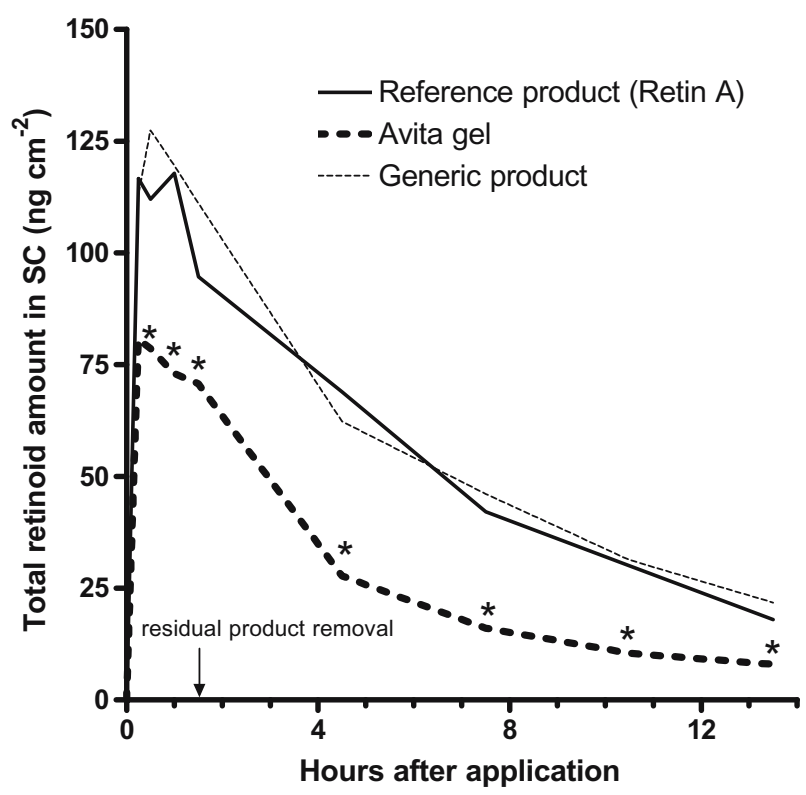

Fig. 4. DPK profiles (means of $n=49)$ of three $(0.025 \% \mathrm{v} / \mathrm{v})$ tretinoin gel products in human stratum corneum $(S C)$. Solid line=reference product $($ Retin- $A)$; dotted line=generic formulation; dashed line $=\mathrm{A}$ vita gel (redrawn from (72)). At the time points indicated with an asterisk (*), a significant difference between Avita and both the reference and gel products exist (analysis of variance, Fisher's protected least significant difference, $p<0.05)$. moment, these uncertainties mean that the minimum number of subjects required for a bioequivalence study cannot be specified.

Another, but more invasive (and probably less acceptable) option is to use a procedure which removes all the SC at once. In this case, cyanoacrylate adhesive is applied to a glass slide which is then pressed firmly to the skin for about $30 \mathrm{~s}$. Subsequent removal in one quick movement removes essentially all of the SC in one complete sheet $(77,78)$. However, validation of the method for bioavailability/bioequivalence studies has not been reported.

Various approaches to quantify the SC amount removed have been considered but, at this time, no single method has achieved universal acceptance. The most straightforward procedure (and, in fact, the one needed to calibrate all others) is to weigh each tape-strip before and after SC removal and to determine the amount $(m)$ of tissue removed from the difference between these weights. As the area $(A)$ of SC stripped is known, and the density $(\rho)$ of the tissue has been published (5), it is possible to convert the SC weight into a depth, or distance $(x)$, into the barrier: $x=m /(A \cdot \rho)$. Additional standardization is then possible by including in the experimental design, concomitant measurements of transepidermal water loss (TEWL) during the tape-stripping process (this can be performed either at the drug product application site, or at a position adjacent to the treated skin when the formulation contains volatile or occlusive excipients that can interfere with the TEWL measurement). Baseline TEWL $\left(\mathrm{TEWL}_{0}\right)$ across unstripped SC of thickness $L$ is given by Fick's first law of diffusion:

$$
\mathrm{TEWL}_{0}=\frac{D \cdot K}{L} \Delta C
$$

where $D$ and $K$ are the diffusion coefficient of water in the $\mathrm{SC}$, and the SC-viable tissue partition coefficient of water, respectively, and $\Delta C \sim 1 \mathrm{~g} \cdot \mathrm{cm}^{-3}$. After tape-stripping has removed a depth $x$ of SC, the TEWL will have increased to a new value given by:

$$
\operatorname{TEWL}_{x}=\frac{D \cdot K}{(L-x)} \Delta C
$$

Inversion of this second expression yields a linear relationship between $\left(\mathrm{TEWL}_{x}\right)^{-1}$ and $x$, and the intercept of this line on the $\mathrm{x}$-axis equals the SC thickness $(L)$ (Fig. 5 (79)).

With this information in hand, all SC stripping data can now be expressed, not in terms of "amount per ten strips," or even as amount per volume of SC, but as an amount per normalized fraction of SC removed $(x / L)$, a strategy which allows results from disparate subjects of different SC thicknesses to be rationalized (Fig. $6(80)$ ). While this appears to be a sensible, general solution to the problem, it is important to point out one caveat: as the tape-stripping procedure and the TEWL measurements require a finite amount of time, analysis of the data for rapidly permeating compounds may be complicated by the fact that the time to obtain the tapestrips, and to record the changes in TEWL, may be similar to the application time, and the drug's concentration gradient may therefore shift during the data collection process (65).

Other methods to quantify the SC removed by tapestripping have been proposed and demonstrated. Clearly, the 

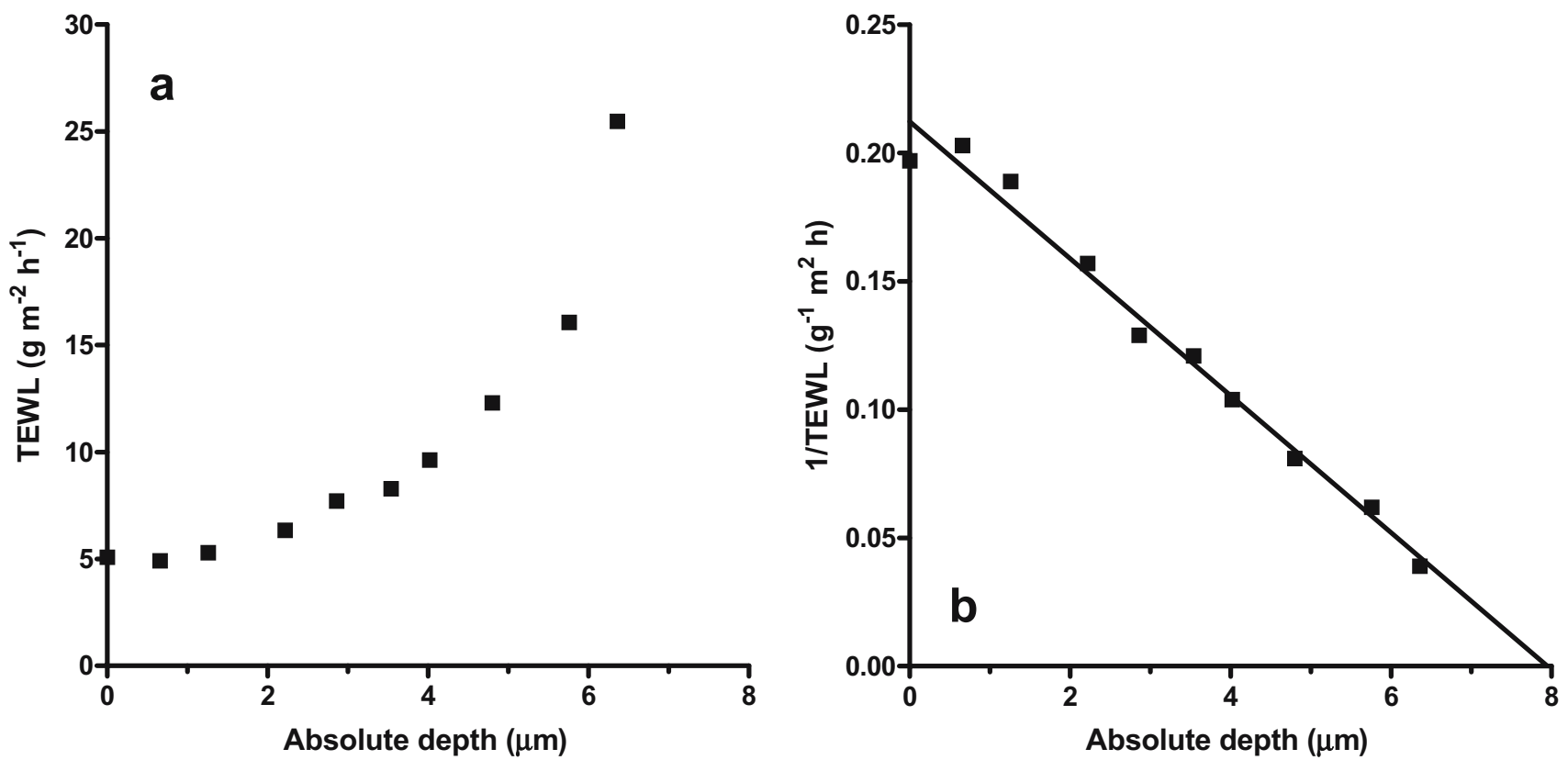

Fig. 5. a Progressive increase of transepidermal water loss $(T E W L)$ as a function of the absolute depth $(\mu \mathrm{m})$ of stratum corneum removed. b The data in a, re-plotted as a linear relationship between (TEWL) ${ }^{-1}$ and the depth of SC removed. The x-axis intercept equals the SC thickness.

gravimetric approach is not without problems (static electricity on the tapes, misleading weights due to uptake of formulation excipients, small amounts of SC removed, tediousness, etc.) and a more direct assessment would be useful. Progress has been made both with respect to the use of a protein assay to quantify keratin on the tapes, and via a spectrophotometric method evaluating the scattering/absorbance of UV/visible radiation; the latter procedure also has the potential to quantify drug on the tape-strip, assuming that an absorbance band distinct from those of the SC can be identified. Both techniques have the potential to be more 'high-throughput' once sufficiently optimized; however, for the moment, neither approach has been perfected, rejection of individual data has not been eliminated, and both rely upon the 'gold standard' weighing measurement for calibration (81-86). Another option, which deserves a final word in this context, is the application of infrared spectroscopy (IR), particularly its use in vivo via the attenuated total reflectance (ATR) mode. In this approach, it is possible to semiquantitatively assess an IR-active drug (assuming there is at least one absorbance within its IR spectrum distinct from those in that of the SC) either on a series of tape-strips or in the outermost micron or so of SC which has not yet been stripped from the skin (44,45,87-91) (Fig. 7).

The method has often been applied to a model permeant, 4-cyanophenol (the $\mathrm{C} \equiv \mathrm{N}$ (nitrile) absorbance from which is particularly intense and occurs at a frequency where the SC is essentially IR-transparent (64-67)), and also to other more relevant substances such as the anti-fungal drug, terbinafine (45). The IR spectrum of SC also contains information about the amount of endogenous lipid and protein present as a function of depth into the barrier (92), although this feature has not been explored as a potentially simple approach to quantify the amount of tissue removed on each tape-strip. Recent developments in IR imaging coupled with ATR, and the application of chemometrics to better "tease out" spectral information, may prove to be useful avenues for further research in this regard (93). However, even supposing the existing limitations can be overcome, the principal weakness of IR remains: namely, its relative insensitivity compared (for example) to analysis of extracted tape-strip samples by HPLC.

\section{Interpretation of Tape-Strip Data}

When drug and SC are quantified on each tape-strip removed post-treatment of the SC, and TEWL measurements have been made to determine SC thickness, it is possible to display the concentration of the permeant $\left(C_{x}\right)$ as a function of its relative depth $(x / L)$ into the barrier (Fig. 8). Assuming Fickian diffusion and the following boundary conditions:

- the 'dose' of the drug applied is infinite-that is, the drug concentration in the vehicle $\left(C_{v}\right)$ remains constant throughout the application time $(t)$

- the SC initially contains no drug $\left(C_{x}=0\right.$ at $\left.t=0\right)$

- the viable skin provides a 'perfect sink' for the penetrating drug $\left(C_{x}=0\right.$ at $x=L$ for $\left.t \geq 0\right)$

then the profile is given by $(46,64,94)$ :

$$
\begin{aligned}
C_{x}= & K \cdot C_{v} . \\
& \left\{(1-x / L)-2 / \pi \sum_{n=1}^{\infty} 1 / n \sin (n \pi \cdot x / L) \cdot \exp \left(-D \cdot n^{2} \cdot \pi^{2} \cdot t / L^{2}\right)\right\}
\end{aligned}
$$

where $K$ is the SC-vehicle partition coefficient of the drug and $D$ is its diffusivity through the barrier. When data like those in Fig. 8 are fitted with the above expression, it is possible to derive two parameters, $K$ and $D / L^{2}$, which 


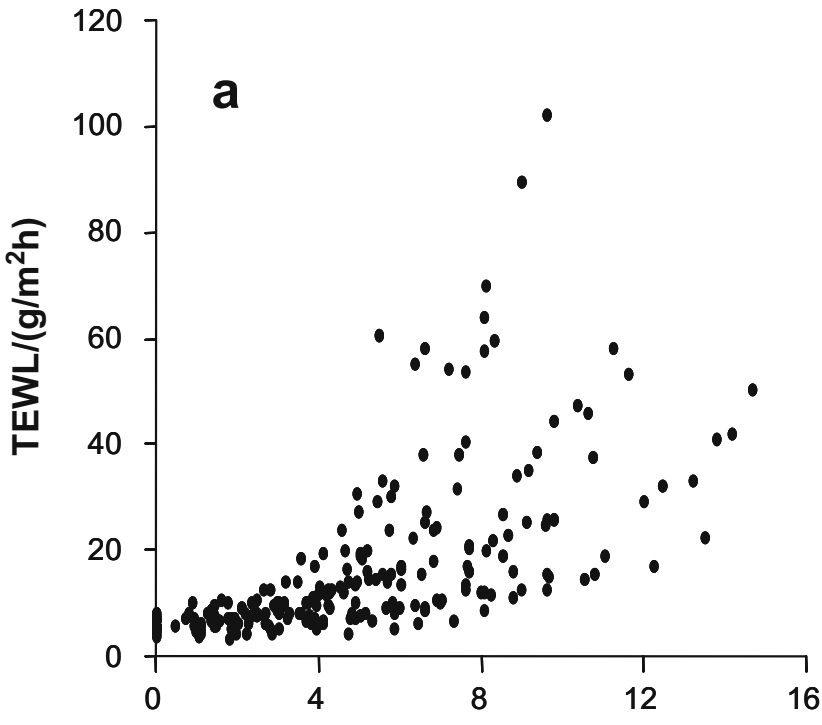

SC Thickness Removed (x) / $\mu \mathrm{m}$

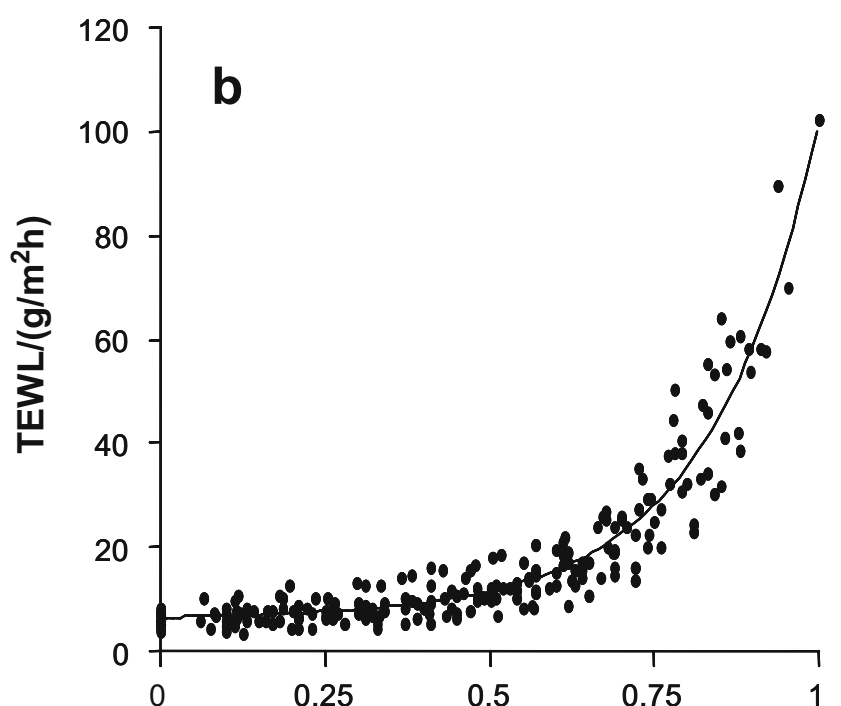

Normalized SC Thickness Removed (x/H)

Fig. 6. a Increase in transepidermal water loss (TEWL) as a function of the amount of SC thickness removed ( $x$ ) by serial tape-stripping $(n=20)$. b Increase in TEWL as a function of SC thickness removed $(x)$ normalized by the corresponding intact membrane thickness $(H)$ for each individual.

characterize drug uptake into the SC from the applied formulation. These physicochemical parameters are relevant to DPK in that they report, respectively, on the extent and the rate of drug uptake into the SC: the partition coefficient $(K)$ reflects the relative affinity of the drug for the SC compared to the applied formulation and is related to the extent of absorption, therefore; the ratio $D / L^{2}$ has units of (time $)^{-1}$ and can be considered as a first-order rate constant for drug transport through the $\mathrm{SC}$-a measure of absorption rate, in other words. Integration of the concentration profile equation yields an "area-under-the-curve" (AUC (46)):

$$
\begin{aligned}
\mathrm{AUC} & =\int_{0}^{1} C_{x} d(x / L) \\
& =K \cdot C_{v}\left\{\frac{1}{2}-\frac{4}{\pi^{2}} \sum_{n=0}^{\infty} \frac{1}{(2 n+1)^{2}} \exp \left(-\frac{(2 n+1)^{2} \cdot \pi^{2} \cdot D \cdot t}{L^{2}}\right)\right\}
\end{aligned}
$$

which represents the total amount of drug in the SC at the end of the application period $(t)$. Together, $K, D / L^{2}$ and AUC

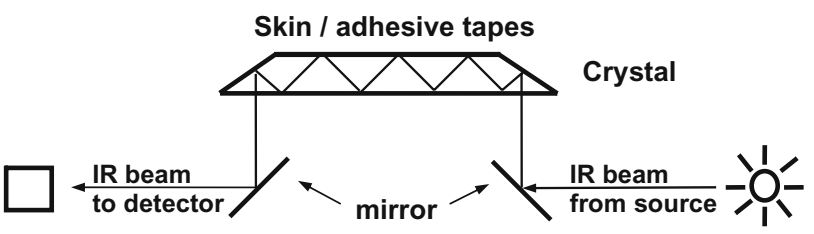

Fig. 7. Schematic diagram of an attenuated total reflectance (ATR) device mounted on a Fourier transform infrared (FTIR) spectrometer. The sampling area of the crystal is made to contact with the treated skin site or the tape-strip. IR radiation from the spectrometer is deflected by a first mirror to the ATR crystal, in which it undergoes total internal reflection, penetrating superficially into the skin, before exiting the crystal and being re-directed to the detector. offer DPK parameters that can be used to objectively compare topical drug bioavailabilities from different formulations. They also allow differences in bioavailability to be explained mechanistically; for example, to answer the question whether a putative penetration enhancer elicits improved drug delivery by increasing partitioning into, or by facilitating diffusion
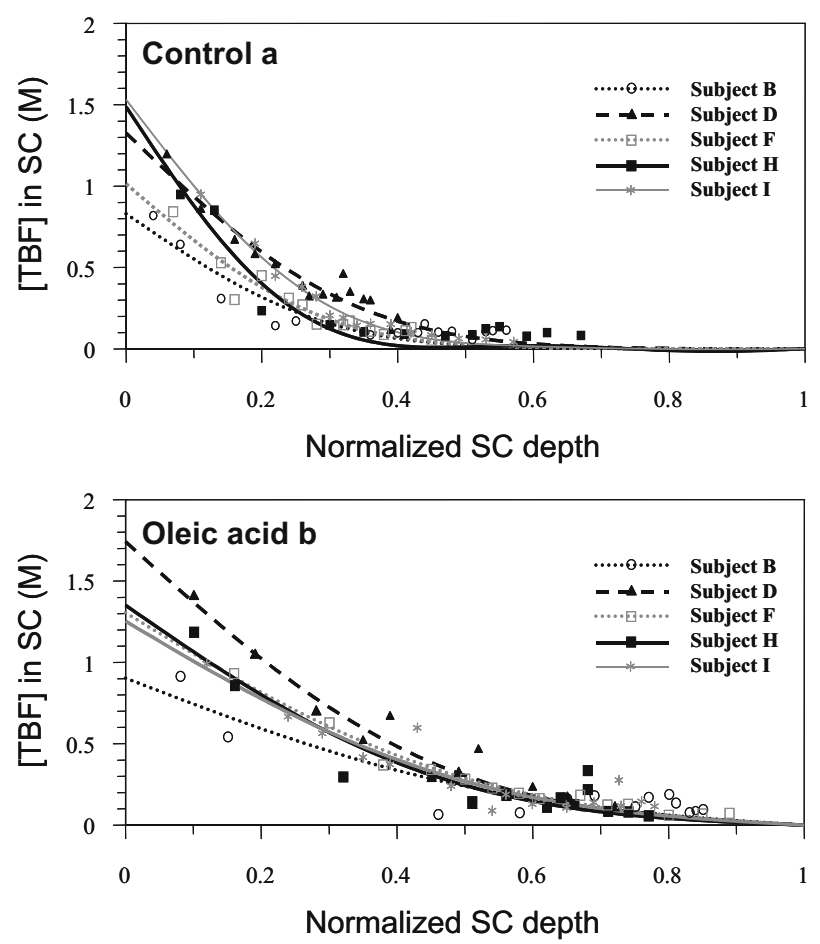

Fig. 8. Terbinafine $(T B F)$ concentration versus $\mathrm{SC}$ depth profiles in vivo $(n=5)$. The individual data points and the best fits of Eq. 1 to the results are shown following application a in the control formulation (EtOH-IPM, 50:50), and $\mathbf{b}$ in the same formulation containing 5\% oleic acid. Redrawn from (46). 
through, the barrier. This capability has been illustrated using the anti-fungal drug, terbinafine (Fig. 8 (44)).

Finally, it is worth pointing out that once $K$ and $D / L^{2}$ have been evaluated from an experimentally determined $C_{x}$ versus $x / L$ profile, they can be used with the equation above to predict AUC at all other times of application $(t)$. Providing that the parameters do not change with time [which, it must be admitted, is not always the case (45)], this means that the complete absorption profile of the drug can be derived from a relatively short-duration experiment. Again using terbinafine, some preliminary validation of this idea has been achieved [Table I (46)]. A more extensive effort, with ibuprofen as the delivered drug, has confirmed this earlier finding (94). In addition, this data analytical approach has value with respect to experimental design; with knowledge of $D / L^{2}$, the classic lag-time for diffusion $\left(L^{2} / 6 D\right)$ across the $\mathrm{SC}$ can be calculated, as well as the time necessary to reach steadystate transport $\left(\sim 2.7 \cdot L^{2} / 6 D(95)\right)$. This information should permit more efficient DPK studies to be performed with tape-stripping timed to provide the maximum possible discrimination, for example, between the different drug products under evaluation.

\section{Tape-Stripping-Unresolved Issues}

Despite the clear value of a DPK approach based upon tape-stripping, there remains work to be done before its usefulness can be fully exploited. First and foremost, the method has to be validated appropriately via clear demonstrations that significant differences in drug uptake into normal SC translates into clinically distinguishable scenarios in the real world; that is, the hypothesis that the SC concentration profile reflects drug availability at the site of action must be confirmed. Logically, this will be more straightforward to accomplish with drugs having their site of action on or in the SC (e.g., anti-fungals), and it is likely that, at least initially, the case will have to be made on a drug class by drug class basis. Although it is clearly expedient to move quickly to an easier approval path for generic topicals, the value and relevance of any approach adopted must be recognized by the dermatologist and must be seen to translate into a measurable clinical outcome.

Second, the procedures to be followed, and the DPK protocol itself, have to be at least partially standardized. Quantification of the SC removed is mandatory and there is an important need to develop a simple and rapid method for

Table I. Comparison Between the Experimentally Determined Values of AUC (mean \pm SD; $n=4$ ) Following 2 and $4 \mathrm{~h}$ of Terbinafine Application, and the Predictions Based Upon $\mathrm{K}$ and $\mathrm{D} / \mathrm{L}^{2}$ Results Determined from the Data Obtained after a 30-min Application

\begin{tabular}{lcc}
\hline Treatment time $(\mathrm{h})$ & $\begin{array}{c}\text { Experimental } \\
\text { AUC } \times 10(\mathrm{M})\end{array}$ & $\begin{array}{l}\text { Predicted } \\
\text { AUC } \times 10(\mathrm{M})\end{array}$ \\
\hline 0.5 & $2.60 \pm 1.09$ & - \\
2 & $4.28 \pm 1.81$ & $4.76 \pm 1.95^{a}$ \\
4 & $4.17 \pm 1.03$ & $5.21 \pm 2.68^{a}$ \\
\hline
\end{tabular}

Data from [45].

${ }^{a}$ Experimental value is not significantly different $(p>0.05)$ from the corresponding predicted result. this purpose. With such knowledge, complete removal of the SC is not necessary for comparative purposes, thereby allowing for a less invasive procedure; further, this means that the method does not have to be restricted to one particular adhesive tape, the choice of which can be based on methodological criteria such as ease of drug extraction, absence of components that may potentially interfere with the drug assay, and allowing removal of the SC with a reasonable number of strips $(\sim 10-15)$. Cleaning the treated area of the SC prior to tape-stripping is important and must be capable of removing excess formulation efficiently without inadvertently 'driving' drug into the barrier. This includes displacement of vehicle from the natural "furrows" in the skin (91), the depth of which can be significantly greater than the SC thickness. Debate continues as to whether one or two tape-strips should be taken initially and discarded, the argument being that any drug lodged on these layers, even after cleaning the skin, would never be absorbed. For the moment, no consensus exists on this point but clearly it will be necessary to better define the procedures to be followed when cleaning the skin at the end of the application period. This issue is particularly important both for poorly penetrating chemicals (e.g., those of higher molecular weight) and for drug products administered in complex vehicles (such as those including liposomes or nanoparticles).

Third, the question of spatial localization of drug must be addressed; specifically, it has been recognized that the target for certain drugs may be a skin appendage (a hair follicle, or a sebaceous gland, for instance) and that particular formulations have been proposed for optimization of delivery to these structures. Exactly how tape-stripping might be used to compare vehicles which set out to achieve, for example, follicular targeting, has not been demonstrated. In this case, it would be necessary to show that SC levels are correlated with drug amounts in the appendage; however, if a formulation did specifically target a follicle, it might be logical to think that one would find less drug in the SC as a result. The problem is complex, therefore, and may need to call upon application of recent work which has attempted to deduce the contribution of follicular transport to total drug delivery by comparing SC uptake in normal skin to that in skin whose follicles have been physically sealed (96-99). Obviously, further validation is required using drugs and drug products designed to act on these appendageal structures.

Finally, there is the question of the relevance of the DPK approach, which has been developed for use on normal skin, to drug performance on diseased skin. In part, this relates to the validation issue discussed above and the need to correlate DPK measurements with clinical outcome. There is also the outstanding unknown as to whether DPK, or a modified version thereof, can be adopted for use on diseased tissue. It is important to acknowledge, furthermore, that the methodology in its present state does not differentiate free and bound drug in the SC.

\section{MICRODIALYSIS}

During the last decade, microdialysis has been shown to be a promising technique for the assessment of cutaneous drug delivery $(100,101)$. The technique is based on the passive diffusion of compounds down a concentration gradi- 

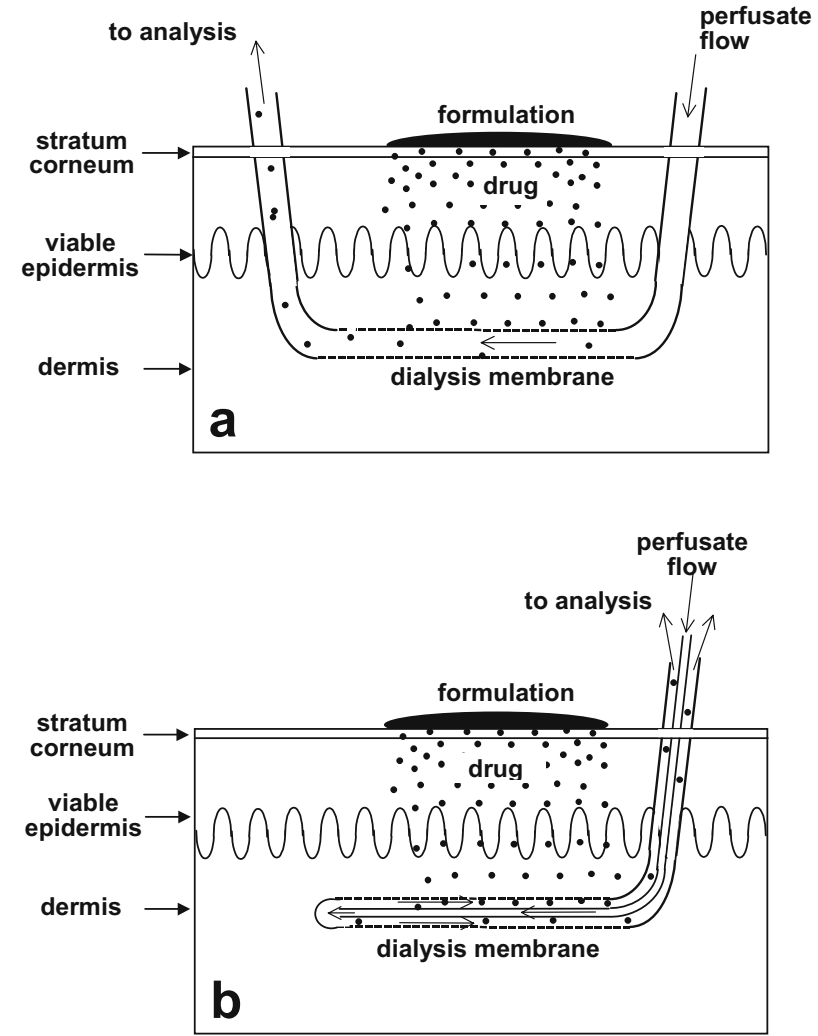

Fig. 9. Schematic diagram of two currently used microdialysis probes for DPK experiments. In both cases, a semi-permeable dialysis membrane collects the absorbed free drug. a Single-lumen probe: the membrane is located in the middle of the probe, and the perfusate flow is mono-directional. b Dual-lumen probe: the membrane is on the tip of the probe, and the perfusate flow is bi-directional.

ent across a semi-permeable membrane forming a thin hollow "tube" (typically a few tenths of a millimetre in diameter), which-at least, in theory-functionally represents a permeable blood vessel. Two kinds of probe are in common use: the so-called linear and concentric probes (Fig. 9). The former is the most prevalent design used because it is simple, thinner and inexpensive to manufacture from artificial kidney fibres (102-104). The probe is implanted superficially into the dermis or subdermis, parallel to the skin surface via a guide cannula. The fibre is slowly perfused with a physiological solution, which equilibrates with the extracellular fluid of the surrounding tissue, exchanging substances smaller than the cut-off value of the membrane. The exchange of material is driven by passive diffusion (105), and the microdialysis technique may be used not only to monitor the extracellular concentrations of exogenous or endogenous compounds, therefore, but also to deliver drugs to a specific target tissue.

A key feature of microdialysis is its ability to continuously monitor the extracellular concentration of a drug in different compartments of the body. Indeed, a microdialysis probe can be implanted in virtually any body organ or tissue (106). Among the limited number of techniques available for the direct assessment of drug concentrations in dermis, cutaneous microdialysis is the only one that allows unbound analytes to be sampled. Hence, microdialysis provides a pharmacokinetic profile, with high temporal resolution, of the drug in the target tissue. As the level of unbound drug generally determines the pharmacodynamic response, the pharmacological relevance of the method is clear (Fig. 10).

The feasibility of microdialysis to sample a drug in the skin has been demonstrated in animals and humans $(101,107)$. Microdialysis has been shown to be useful (a) to assess free drug concentrations in the dermis or subcutaneous tissue, depending on probe depth, after topical application of a formulation, (b) to compare different formulations of a drug and assess bioequivalence/bioavailability, and (c) to measure whether therapeutic concentrations are reached in the skin.

Another important advantage of microdialysis is that no biological fluid is removed. In vivo, in man, multiple sampling sites can be simultaneously set up and used to determine, for example, drug penetration into the skin and the effect of different vehicles (107), cutaneous metabolism $(108,109)$, and the impact of barrier disruption (110). This same flexibility has also allowed the impact of probe depth on the data obtained to be assessed $(111,112)$, and has allowed the inherent variability of the method to be reduced (113).

The initial research evaluating microdialysis for topical delivery was mostly performed in rats, an animal chosen for its ease of handling rather than for its suitability as a model for human skin penetration. Proof-of-concept was established with 5-fluorouracil (103), and subsequent work evaluated, inter alia, the impact of formulation, the action of penetration enhancers, and the improved delivery possible with iontophoresis $(111,112,114-122))$. A significant effort has also been made to correlate microdialysis recovery with barrier perturbation. Using salicylic acid as a model penetrant, uptake was shown to be proportional to barrier function as measured, for example, by transepidermal water loss (123), suggesting that the technique might be useful for assessing bioavailability in topical dermatoses.

In humans, the first applications of microdialysis involved the measurement of (a) ethanol absorption (124), and (b) cutaneous glucose levels $(125,126)$. Subsequently, the transdermal delivery of nicotine released from a commercial patch has been investigated $(127,128)$, and some correlation has been found between the plasma concentration and

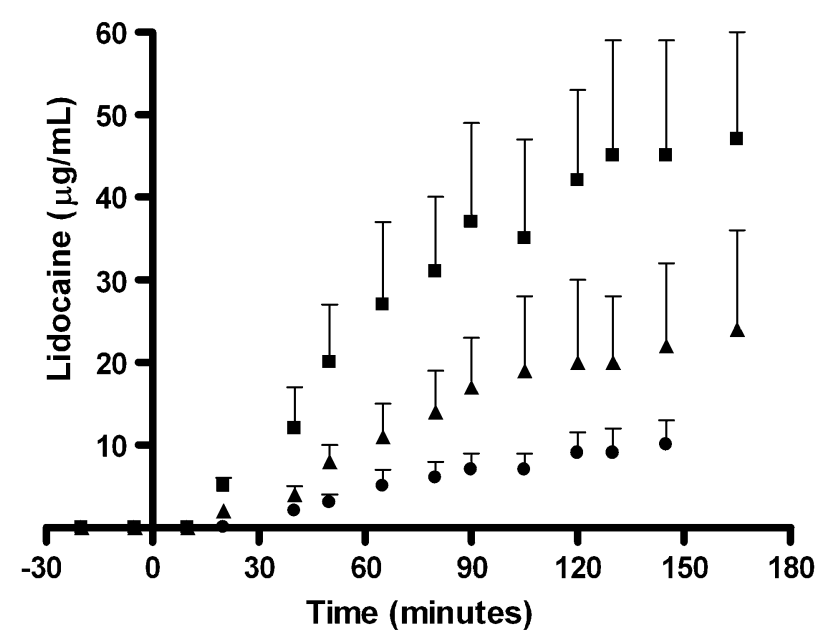

Fig. 10. Penetration of lidocaine from three different formulations into the dermis of eight volunteers measured by microdialysis (from (107)). 
perfusate level versus time profiles. Less success was obtained with estradiol, however, with eight out of ten experiments revealing no detectable estradiol in the dialysate. Likewise, even after barrier disruption, betamethasone 17-valerate, and the highly protein-bound drug, fusidic acid, could not be successfully sampled (129). On the other hand, less hydrophobic and more quickly penetrating substances, such as non-steroidal anti-inflammatory drugs, have been monitored easily by cutaneous microdialysis (108,109,111, 130-132). The cutaneous penetration of salicylic acid through normal and perturbed skin using microdialysis sampling has been shown to be more sensitive than non-invasive bioengineering techniques in detecting the effect of acetone treatment. A positive dose-response was also found between salicylic acid penetration and the degree of irritant dermatitis induced by sodium lauryl sulphate pre-treatment.

The skin penetration of various salicylate esters and their skin metabolism in dermal and subcutaneous tissue has been studied (109). The delivery of topical diclofenac was similarly demonstrated following a single application of a gel (130). The topical bioequivalence of lidocaine delivery from a microemulsion and from a conventional oil-in-water emulsion was assessed pharmacokinetically by microdialysis and the results were compared to pharmacodynamic measurements as well (107). Furthermore, microdialysis has been used to study iontophoretic delivery of propanolol, acyclovir and flurbiprofen $(111,133,134)$. Additionally, the role of stratum corneum, and of dermal microvasculature perfusion, in the penetration and tissue distribution of the water-soluble drugs, acyclovir and pencyclovir, have been investigated by cutaneous microdialysis (135).

Microdialysis is claimed to be minimally invasive, provoking only a minor, reversible trauma caused by insertion of the guide cannula when implanting the probe (136-138). Moreover, changes in the tissue surrounding the probe may affect the recovery and measurement of the analyte. Therefore, when using the microdialysis technique, an equilibration of $60-90 \mathrm{~min}$ following probe insertion is usually an integral part of the protocol $(136,137)$. The effects of the probe insertion can be classified as (a) direct trauma to cells and tissues; (b) modification of blood perfusion due to an axon reflex; and (c) inflammatory or "foreign body" reactions to the probe (139). In vivo assessment of the skin after insertion of the microdialysis probe is usually performed using non-invasive measurements of TEWL, cutaneous blood flow, and histology (136-139). The extent and duration of "trauma" to the skin can be modulated by the probe design and the use of a local anaesthetic. Guide cannulas with smaller dimensions generally provoke smaller reactions and the provoked increases in local blood flow (and histamine release) subside more quickly; the concomitant use of a topical anaesthetic cream can reduce the duration of vascular response to less than an hour (140-142). Longer-term implantation has been studied in animal models and a general inflammatory response observed: infiltration of lymphocytes at $8 \mathrm{~h}$, cell changes and cell attachment to the dialysate membrane at $24 \mathrm{~h}$, fibrosis after a week, and scar tissue formation. However, apart from the initial reaction on probe insertion, tissue responses are relatively minor throughout the duration of a typical microdialysis experiment $(\sim 8 \mathrm{~h}(103,138,143))$.

\section{Method Development and Optimization}

Since microdialysis operates under non-equilibrium conditions, the concentration of analyte in the dialysate is lower than that in the extracellular fluid surrounding the probe. Relative recovery has been defined as the ratio of these concentrations, and should be independent of the absolute, free concentration of the compound in the skin (assuming no binding to the dialysis membrane). Several approaches have been suggested for determination of the in vivo recovery of the analyte by the microdialysis probe: the stop-flow method (144), the extrapolation-to-zero flow technique (142), the point of no net flux (102), and retrodialysis (145). Extensive reviews of the various techniques have been published (105). Retrodialysis is probably faster and more convenient than the other alternatives. This, "internal reference technique" $(146,147)$, introduces a marker into the perfusate and the rate of delivery of this compound is measured during the microdialysis experiment. Recovery $(R)$ is then determined from:

$$
R=100 \bullet\left(C_{\text {in }}-C_{\text {out }}\right) / C_{\text {in }}
$$

where $C_{\text {in }}$ is the concentration of marker perfused into the fibre and $C_{\text {out }}$ is the concentration determined in the sample. This method assumes, and/or demonstrates, that the recovery efficiency of the target analyte is quantitatively similar to the delivery of the retrodialysis marker. It follows that the marker should physicochemically resemble, as far as possible, the analyte of interest. An important advantage is that fluctuations in the recovery of the probe during the experiment are taken into account by continuous retrodialysis of the marker. Conversely, a significant limitation is that the presence of the marker perturbs in some way (e.g., competition for binding) the local disposition of the drug.

The relative recovery is influenced by the probe, the tissue perfused and the analyte of interest (100). To increase the relative recovery, the length of the probe and its molecular weight cut-off limit should be increased, while perfusate flow should be reduced. Typically, in dermal microdialysis, the probe length is $\sim 1-2 \mathrm{~cm}$, and the flow rate between 0.5 and $5 \mu \mathrm{l} / \mathrm{min}$. The molecular weight cut-off chosen is typically in the range of 5-20 KDa to avoid protein leakage into the dialysate. For dermal microdialysis, the linear probe design is preferred whereas, for subcutaneous implantation, both linear and concentric probes have been used.

Because the dialysis fibre excludes diffusion of proteins, the samples are sufficiently purified for further analysis. The principal challenge to the procedure is sensitivity, especially for the measurement of (a) lipophilic permeants which are slowly and poorly extracted into the microdialysis perfusate, and (b) compounds with high protein binding. For example, in earlier work, fusidic acid and betamethasone-17-valerate were not detectable by microdialysis (129). Although it has been suggested that recovery may be improved by introducing co-solvents (e.g., polyethylene glycol), or cyclodextrins, or lipids, into the perfusate, or by changing its $\mathrm{pH}$, the advantages of such a strategy must be balanced against potential tissue alterations and their impact upon biocompatibility $(148,149)$. Low rates of perfusion $(0.5-5 \mu 1 / \mathrm{min})$ are mandatory for compounds with low recoveries, especially those which are highly protein-bound. Nevertheless, this 
means that the samples are of low volume and contain small absolute amounts of analyte. The analytical challenge is therefore considerable and demands sensitive techniques; HPLC may not be sufficient and the use of specific biosensors and mass spectrometry (as well as other techniques) has been reported $(138,150)$.

To validate the use of microdialysis, as a method to assess bioavailability/bioequivalence, a systematic comparison with existing methods is essential. Generally speaking, when different formulations of a drug have been compared, microdialysis has determined the same rank order as other approaches. However, the absolute, measured amounts of drug absorbed are different (Fig. 11). For example, the quantity of drug found in the skin after an in vitro diffusion cell experiment is higher than that detected by microdialysis (103). The explanation for this observation is that the classic in vitro technique (a) measures both bound and unbound drug, and (b) does not reproduce the effects of local blood flow on absorption.

A particularly positive, and rather unique feature, of microdialysis is that the method can be used to evaluate drug permeation across both normal and diseased skin. Furthermore, in the same psoriatic patient, penetration at a specific lesion may be compared to that through unaffected skin. In addition, as well as measuring drug concentration, microdialysis may also have the potential to quantify a biomaker, or the concentration of another surrogate measure, for therapeutic activity. Concomitant pharmcokinetic and pharmacodynamic evaluations are therefore possible.
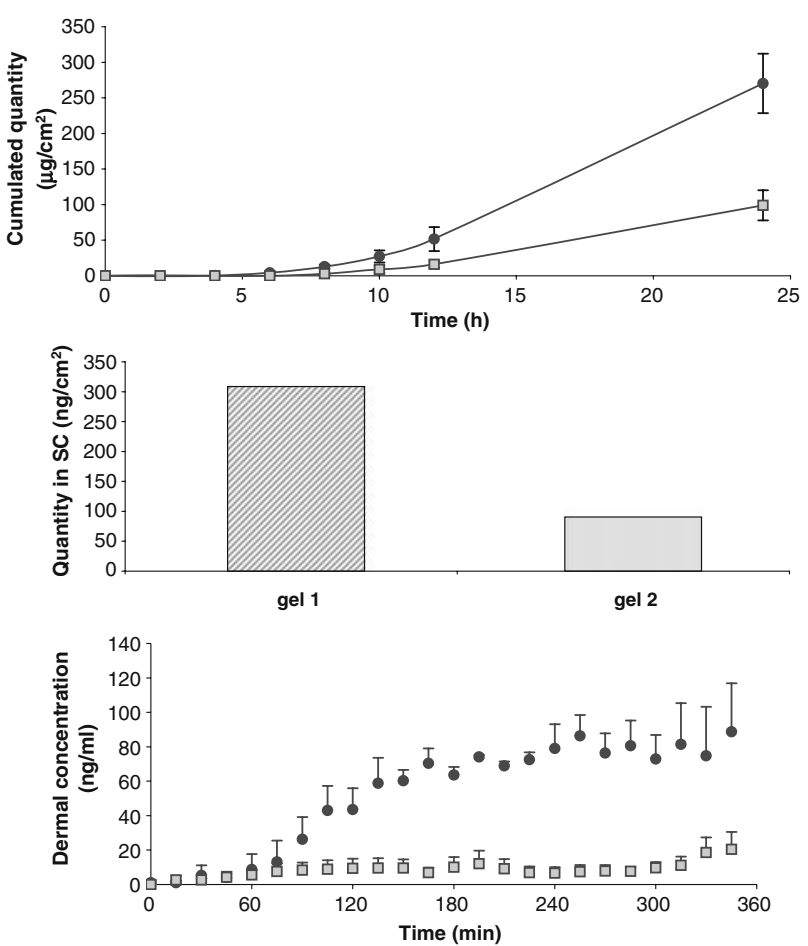

Fig. 11. Local availability of ketoprofen applied topically from two gel formulations: assessment $\mathbf{a}$ in vitro in diffusion cells (upper panel), $\mathbf{b}$ in vivo by tape stripping (middle panel), and $\mathbf{c}$ by dermal microdialysis (lower panel; A.-R. Denet and V. Préat, unpublished data).

\section{Interpretation of Microdialysis Data}

It should be emphasized that data from microdialysis experiments can be analyzed to yield practical pharmacokinetic parameters. $C_{\max }, T_{\max }$, absorption constant, lag time and AUC can be determined from the plot of free drug concentration in the dermis as a function of time. For example, a one-compartment model has been used to differentiate between results from two formulations of lidocaine (107) via comparison of absorption rates and lagtimes. In this case, prilocaine was used as a retrodialysis calibrator. In another elegant study, cutaneous microdialysis demonstrated that the skin/plasma ratio of 8-methoxypsoralen was orders of magnitude greater following topical administration of the drug (as compared to oral dosing). Evaluation of $C_{\max }$ in the skin, furthermore, permitted optimization of the time of UV irradiation in PUVA therapy (151). Elsewhere, topical application of the antifungal fluconazole resulted in free dermal concentrations below the minimal inhibitory concentration (112). This ability to measure whether a therapeutic concentration of a drug in the skin has been attained after topical administration is a particular advantage of microdialysis. In addition, both the applied drug and any metabolites formed by biotransformation in the skin are, in theory, detectable by this method. However, only in a few exceptional circumstances [e.g., salicylate derivatives (109)] has significant metabolism been observed.

The number of subjects necessary for a microdialysis study to determine whether two formulations are bioequivalent depends on the degree of variability observed in the data. From a practical standpoint, it has been suggested that this problem should be tackled by ensuring that each subject acts as its own control (113). Under these circumstances, and performing duplicate measurements in every volunteer, $\sim 20$ subjects are considered sufficient to distinguish two formulations within the classic $80-125 \%$ confidence interval. In contrast, most microdialysis bioequivalence studies in the literature have been performed with six to ten volunteers $(107,110)$.

\section{Microdialysis-Unresolved Issues}

While many studies comparing microdialysis to other methods have shown reasonable correlation, further validation work is essential if this approach is to be recognized by the regulatory agencies as an option for bioavailability and bioequivalence assessment. One key issue in the development of a standardized protocol is the reproducible insertion of the microdialysis probe to a consistent depth within the skin. Probe implantation remains a technical challenge that is only mastered with patience and practice. Although the significance of probe depth on the resulting permeation profile is a matter of some debate, it is clear that implantation in the either the superficial dermis, or the deep dermis or the subcutaneous tissue can have an obvious impact on the data obtained $(111,112)$.

A further limitation to the routine application of microdialysis, at the present time, is analysis of the very low levels of drug in the dialysate. The most successful demonstrations of the approach have been made with compounds which readily penetrate the skin and which have decent aqueous solubilities and low protein binding. Such molecules are rare, 
however, and are not always relevant to dermatological therapy. Corticosteroids, on the other hand, the vasoconstriction response to which might offer a useful validation 'marker' for microdialysis, have never been successfully monitored by the technique (presumably due to their low permeabilities, poor aqueous solubilities and high protein binding).

In addition, even though microdialysis may be reasonably termed "minimally invasive," its use in vivo is typically limited to experiments lasting 5-10 h. For slowly permeating drugs, therefore, the quantities absorbed in this time will be minimal, and the analytical problems quite daunting.

\section{OTHER APPROACHES}

Finally, a few words about some alternative strategies, of which only confocal Raman spectroscopy is receiving significant attention at this time.

\section{Skin Biopsy}

Skin biopsies, whether to the level of the dermis (shave biopsy) or through to the sub-cutis (punch biopsy), are invasive and generally performed under local anaesthesia. While such methods have a place in dermatological surgery (to remove warts and small tumours, for example), their application for tissue sampling and analysis post-drug application has not attracted much attention as a routine approach for use in vivo. Despite the obvious advantage of offering a 'snapshot' of drug disposition in the different skin layers, the aggressive nature of the biopsy rules out any chance that it might be adopted as a standard procedure. Even attempts to minimize tissue trauma $(131,152)$ fail to render the method remotely acceptable and its use in the foreseeable future will be restricted to animal and in vitro studies.

\section{Suction Blister}

Applying a partial negative pressure to the skin disrupts the epidermal-dermal junction and forms a blister which fills progressively with interstitial fluid and serum $(153,154)$. This liquid offers a pharmacokinetic "compartment," therefore, in which a previously applied drug can be sampled with a hypodermic needle and quantified; if multiple blisters are raised (as is possible with certain commercially available devices), then a concentration-time profile of the drug in the skin can be obtained. While on the surface attractive, this approach is also quite invasive and causes obvious scarring, albeit over relatively small areas of skin. The technique can be used to compare topical formulations in a reasonably objective way $(155,156)$, but the potential binding of the drug to skin tissue, especially for very lipophilic species, may mean that very low levels are present, if at all, in the blister fluid (157). Taken together, this has meant that the procedure has not been widely used and, once again, is presently viewed as too invasive for practical and routine application in topical bioavailability experiments.

\section{Follicle Delivery}

Drug delivery to the follicle following application of formulations to the skin is an established fact. Although the follicles occupy a relatively small fractional area (on average, $\sim 0.1 \%$ ) of the surface, it appears that diffusion via these appendages is fast relative to that through intact SC, and probably accounts for the first drug molecules which penetrate deep into the skin (158). Furthermore, the follicle is a bona fide target for skin diseases, such as acne and alopecia, and this raises the issue of drug bioavailability to this specific structure. For example, it has been claimed that certain particle sizes are optimal for sequestration in the follicle (159) thereby concentrating the drug at this site and subsequently providing a sustained release to the local environment. However, a method, with which to quantify such 'targeting', has been difficult to validate unequivocally and no approach can be claimed to have demonstrated practical utility for quantitative bioavailability/bioequivalence assessment to the follicle. Nevertheless, there have been some innovative ideas reported in the literature $(77,160,161)$ and, more recently, somewhat simpler methodology has been described, with which the contribution of follicular transport to the overall permeation of a drug in vivo in man has been estimated $(99,162)$.

\section{Confocal Raman Spectroscopy}

This technique uses the confocal principle to noninvasively examine the outer layers of the skin by Raman spectroscopy. In this way, it is possible to acquire rapidly, in

Table II. Methods to Assess Drug Penetration into and/or Across the Skin

\begin{tabular}{|c|c|c|c|c|c|}
\hline & Method & Measure & Measurement site & $\begin{array}{l}\text { Temporal } \\
\text { resolution }\end{array}$ & $\begin{array}{l}\text { Technical } \\
\text { simplicity }\end{array}$ \\
\hline In vitro & Diffusion cell & Q & $\begin{array}{l}\text { Transport into and } \\
\text { across skin }\end{array}$ & ++ & + \\
\hline \multirow{5}{*}{$\begin{array}{l}\text { In vivo: non- or } \\
\text { minimally invasive }\end{array}$} & Tape stripping & $\mathrm{Q}$ & Stratum corneum & 0 & + \\
\hline & ATR-FTIR & $\mathrm{Q}$ & Stratum corneum & + & + \\
\hline & Raman & $\mathrm{Q} / \mathrm{L}$ & Upper skin & + & + \\
\hline & Microdialysis & $\mathrm{Q}($ free $)$ & Dermis (or subdermis) & ++ & - \\
\hline & Vasoconstriction & A & Microcirculation & + & \pm \\
\hline \multirow[t]{3}{*}{ In vivo: invasive } & Blister & $\mathrm{Q}$ & Extracellular fluid & 0 & \pm \\
\hline & Biopsy & $\mathrm{Q}$ & Skin & 0 & + \\
\hline & Biopsy & $\mathrm{Q}+\mathrm{L}$ & Skin (depth) & 0 & \pm \\
\hline
\end{tabular}


vivo in humans, Raman spectra of the skin as a function of depth (163). The method is able to provide, for example, information on the hydration of the SC, and the manner in which the water concentration profile across the barrier may be altered by the use of moisturizing agents (163-165). Other analytes, such as urea, can also be examined via their unique Raman signature which allows them to be differentiated from the spectrum of the skin's constituents (166).

The approach is comparable to the application of reflectance infrared spectroscopy described earlier. In distinct contrast, however, confocal Raman does not require progressive stripping of the SC to determine the concentration versus depth profile of a target substance, and this is clearly an important advantage. Like ATR-IR, however, confocal Raman requires that the molecule of interest be present at a sufficient concentration, and possess spectral features of sufficient intensity, to permit its differentiation from those of the skin (163). Therein lies, at present, the major drawback of these IR-based techniques. With confocal Raman, in addition, only relative concentrations (rather than absolute levels) can be determined. It remains to be seen, therefore, whether this significant technological development will contribute eventually to the evaluation of topical drug bioavailability and/or bioequivalence. For the moment, the method offers a unique research tool and a novel approach to evaluate skin moisturization.

\section{CONCLUSIONS}

Despite pressure on regulatory agencies, such as the FDA, there is no generally accepted method with which to evaluate the bioavailability and bioequivalence of topical drug products. For the present, therefore, with the exception of the vasoconstriction assay for corticosteroids, and despite the diversity of efforts which have been made (Table II), clinical studies are obligatory. Unlike oral administration, for example, where the blood level of a drug is a generally accepted 'surrogate' for its concentration at the site of action, topical drug delivery poses a more complex problem. In this case, the site of action (even when it is known, which is not always the case) is not always accessible and a suitable 'surrogate', e.g., the SC, has not been generally validated. Two approaches are under most intensive scrutiny: one, microdialysis, strives to make measurements of the drug in a true "biophase" of the skin; the other, tape-stripping, attempts to establish the SC as the 'surrogate' sampling site. In both instances, significant progress has been made and proof-of-concept demonstrated. However, the next steps, i.e., optimization of the procedure(s) and transition to a useful and practical regulatory test, remain challenges for future work.

\section{ACKNOWLEDGEMENTS}

Financial support was provided by Galderma S.A., SMB Galephar, Leo Pharmaceutical Products, Fonds national de la recherche scientifique (Belgium), la Région wallonne (Belgium) and the US Food and Drug Administration. The comments, suggestions, discussions and criticisms of Drs. Christopher Hensby and Vinod Shah, and of Professors
Jonathan Hadgraft, Roger Verbeeck and Annette Bunge, have contributed enormously to the evolution of our thinking about topical bioavailability over the past several years.

\section{REFERENCES}

1. A. Finlay and R. Marks. Determination of corticosteroid concentration profile in the stratum corneum using the skin surface biopsy technique. Br. J. Derm. 107 Suppl 22: 23 (1982).

2. P. M. Elias. Epidermal lipids, barrier function and desquamation. J. Invest. Dermatol. 80:S44-S49 (1983).

3. P. W. Wertz, D. C. Swartzendruber, D. J. Kitko, K. C. Madison, and D. T. Downing. The role of the corneocytes lipid envelopes in cohesion of the stratum corneum. J. Invest. Dermatol. 93:169-172 (1989).

4. P. M. Elias. Epidermal barrier function: intercellular lamellar lipid structures, origin, composition and metabolism. J. Control. Release 15:199-208 (1991).

5. R. L. Anderson and J. M. Cassidy. Variations in physical dimensions and chemical composition of human stratum corneum. J. Invest. Dermatol. 61:30-32 (1973).

6. K. Holbrook and G. Odland. Regional differences in the thickness of the human stratum corneum: an ultrastructural analysis. J. Invest. Dermatol. 62:415-422 (1974).

7. D. Schwindt, K. P. Wilhelm, and H. I. Maibach. Water diffussion characteristics of human stratum corneum at different anatomical sites in vivo. J. Invest. Dermatol. 111:385-389 (1998).

8. M. Fartasch. The nature of the epidermal barrier: structural aspects. Adv. Drug Deliv. Rev. 18:273-282 (1996).

9. N. A. Monteiro-Riviere. Introduction to histological aspects of dermatotoxicology. Microsc. Res. Tech. 37:171 (1997).

10. S. Borsadia, A. H. Ghanem, Y. Seta, W. I. Higuch, G. L. Flynn, C. R. Behl, and V. P. Shah. Factors to be considered in the evaluation of bioavailability and bioequivalence of topical formulations. Skin Pharmacol. 5:129-145 (1992).

11. C. R. Behl, H. Char, S. B. Patel, D. B. Metha, D. Piemontese, and $\mathrm{A}$. W. Malick. In vivo and in vitro skin uptake and permeation studies: critical considerations and factors which affect them. In V. P. Shah and H. I. Maibach (eds.), Topical Drug Bioavailability, Bioequivalence, and Penetration, Plenum, New York, 1993, pp. 225-252.

12. R. Marks and P. Dykes. Plasma and cutaneous drug levels after topical application of piroxicam gel: a study in healthy volunteers. Skin Pharmacol. 7:340-344 (1994).

13. R. O. Potts and R. H. Guy. Predicting skin permeability. Pharm. Res. 9:663-669 (1992).

14. D. Dupuis, A. Rougier, R. Roguet, and C. Lotte. The measurement of the stratum corneum reservoir: a simple method to predict the influence of vehicles on in vivo percutaneous absorption. Br. J. Derm. 115:233-238 (1986).

15. G. L. Flynn. General introduction and conceptual differentiation of topical and transdermal drug delivery systems. Differentiation with respect to delivery kinetics. In V. P. Shah and H. I. Maibach (eds.), Topical Drug Bioavailability, Bioequivalence, and Penetration, Plenum, New York, 1993, pp. 369-392.

16. L. K. Pershing, B. S. Silver, G. G. Krueger, V. P. Shah, and J. P. Skelley. Feasibility of measuring the bioavailability of topical betamethasoe dipropionate in commercial formulation using drug content in skin and a skin blanching bioassay. Pharm. Res. 9:45-51 (1992)

17. A. W. McKenzie and R. B. Stoughton. Method of comparing percutaneous absorption of steroids. Arch. Dermatol. 86:608610 (1962)

18. J. M. Haigh and I. Kanfer. Assessment of topical corticosteroid preparations: the human skin blanching assay. Int. J. Pharm. 19:245-262 (1984).

19. S. Y. Chan and A. Wan PoLi. Quantitative skin blanching assay of corticosteroid creams using tristimulus colour analysis. $J$. Pharm. Pharmacol. 44:371-378 (1992). 
20. L. K. Pershing, L. D. Lambert, V. P. Shah, and S. Y. Lam. Variability and correlation of chromameter and tape-stripping methods with the visual skin blanching assay in the quantitative assessment of topical $0.05 \%$ betamethasone dipropionate bioavailability in humans. Int. J. Pharm. 86:201210 (1992).

21. V. P. Shah. Topical dermatological drug product NDAs and ANDAs-in vivo bioavailability, bioequivalence, in vitro release and associated studies, US Dept. of Health and Human Services, Rockville, 1998, pp. 1-19.

22. L. K. Pershing, S. Bakhtian, G. Poncelet, J. L. Corlett, and V. P. Shah. Comparison of skin stripping, in vitro release, and skin blanching response methods to measure dose response and similarity of triamcinolone acetonide cream strengths from two manufactured sources. J. Pharm. Sci. 91:1312-1323 (2002).

23. J. Johannson and A. Lahti. Topical non-steroidal antiinflammatory drugs inhibit non-immunologic immediate contact reactions. Contact Dermatitis 19:161-165 (1988).

24. M. C. Poelman, B. Piot, F. Guyon, M. Deroni, and J. L. Leveque. Assessment of topical non-steroidal anti-inflammatory drugs. J. Pharm. Pharmacol. 41:720-722 (1989).

25. L. Duteil, C. Queille, M. Poncet, J. P. Ortonne, and J. Czernielewski. Objective assessment of topical corticosteroids and non-steroidal anti-inflammatory drugs in methylnicotinate-induced skin inflammation. Clin. Exp. Dermatol. 15:195-199 (1990).

26. S. Y. Chan and A. Wan PoLi. Quantitative evaluation of druginduced erythema using a tristimulus color analyzer: experimental design and data analysis. Skin Pharmacol. 6:298-312 (1994).

27. P. Treffel and B. Gabard. Feasibility of measuring the bioavailability of topical ibuprofen in commercial formulations using drug content in epidermis and a methyl nicotinate skin inflammation assay. Skin Pharmacol. 6:268-275 (1994).

28. H. Tagami, T. Tadaki, M. Obata, and J. Koyama. Functional assessment of the stratum corneum under the influence of oral aromatic retinoid (etretinate) in guinea-pigs and humans. Comparison with topical retinoic acid treatment. $B r . J$. Dermatol. 127:470-475 (1992).

29. J. W. Fluhr, M. P. Vienne, C. Lauze, P. Dupuy, W. Gehring, and M. Gloor. Tolerance profile of retinol, retinaldehyde and retinoic acid under maximized and long-term clinical conditions. Dermatol. 199 Suppl 1: 57-60 (1999).

30. M. Vershoore, M. Poncet, J. Czernielewki, V. Sorba, and A. Clucas. Adapalene $0.1 \%$ gel has low skin-irritation potential. $J$. Am. Acad. Dermatol. 36:S104-S109 (1997).

31. V. P. Shah. Bioequivalence of topical dermatological dosage forms - methods of evaluation of bioequivalence. Pharm. Res. 15:167-171 (1998).

32. G. K. Menon, K. R. Feingold, and P. M. Elias. Lamellar body secretory response to barrier disruption. J. Invest. Dermatol. 98:279-289 (1998)

33. M. Denda, L. C. Wood, S. Emami, C. Calhoun, B. E. Brown, P M. Elias, and K. R. Feingold. The epidermal hyperplasia associated with repeated barrier disruption by acetone treatment or tape stripping cannot be attributed to increased water loss. Arch. Dermatol. Res. 288:230-238 (1996).

34. P. G. Van der Walk and H. I. Maibach. A functional study of the skin barrier to evaporative water loss by means of repeated cellophane-tape stripping. Clin. Exp. Dermatol. 15:180-182 (1990).

35. C. Pierard-Franchimont and G. E. Pierard. Assessment of aging and actinic damages by cyanoacrylate skin surface strippings. Am. J. Dermatopathol. 9:500-509 (1987).

36. C. Marionnet, F. Bernerd, A. Dumas, F. Verrecchia, K Mollier, D. Compan, B. Bernard, M. Lahfa, J. Leclaire, C. Medaisko, B. Mehul, S. Seite, A. Mauviel, and L. Dubertret. Modulation of gene expression induced in human epidermis by environmental stress in vivo. J. Invest. Dermatol. 121:14471458 (2003).

37. H. Ou-Yang, G. Stamatas, C. Saliou, and N. Kollias. A chemiluminescence study of UVA-induced oxidative stress in human skin in vivo. J. Invest. Dermatol. 122:1020-1029 (2004).
38. H. Wagner, K. H. Kostka, C. M. Lehr, and U. F. Schaefer. pH profiles in human skin: influence of two in vitro test systems for drug delivery testing. Eur. J. Pharm. Biopharm. 55:57-65 (2003).

39. N. Sekkat, Y. N. Kalia, and R. H. Guy. Biophysical study of porcine ear skin in vitro and its comparison to human skin in vivo. J. Pharm. Sci. 91:2376-2381 (2002).

40. J. Inoue and Y. Aramaki. Toll-like recptor 9 induced by tapestripping triggers on effective immune response with $\mathrm{CpG}$ oligodeoxynucleotides. Vaccine 25:1007-1013 (2006).

41. C. Surber, F. P. Schwarb, and E. W. Smith. Tape-stripping technique. In R. L. Bronaugh and H. I. Maibach (eds.), Percutaneous Absorption, Dekker, New York, 1999, pp. 395-409.

42. P. W. Lücker, E. Beubler, W. R. Kukovetz, and W. Ritter Retention time and concentration in human skin of bifonazole and clotrimazole. Dermatologica 169:51-55 (1984).

43. L. K. Pershing, J. Corlett, and C. Jorgensen. In vivo pharmacokinetics and pharmacodynamic of topical ketoconazole in human stratum corneum. Antimicrob. Agents. Chemother. 38:90-95 (1994).

44. I. Alberti, Y. N. Kalia, A. Naik, J. D. Bonny, and R. H. Guy. In vivo assessment of the enhanced topical delivery of terbinafine to human stratum corneum. J. Control Release 71:319-327 (2001).

45. I. Alberti, Y. N. Kalia, A. Naik, J. D. Bonny, and R. H. Guy Effect of ethanol and isopropyl myristate on the availability of topical terbinafine in human stratum corneum in vivo. Int. J. Pharm. 219:11-19 (2001).

46. I. Alberti, Y. N. Kalia, A. Naik, and R. H. Guy. Assessment and prediction of the cutaneous bioavailability of topical terbinafine in vivo in man. Pharm. Res. 18:1472-1475 (2001).

47. P. Clarys, B. Gabard, R. Lambrecht, A. Barel, E. Bieli, and S. Lüdi. There is no influence of a temperature rise on in vivo adsorption of UV filters into the stratum corneum. J. Dermatol. Sci. 27:77-81 (2001).

48. C. Fernandez, F. Nielloud, R. Fortuné, L. Vian, and G. MartiMestres. Benzophenone-3: rapid prediction and evaluation using non-invasive methods of in vivo human penetration. $J$. Pharm. Biomed. Anal. 28:57-63 (2002).

49. S. A. Wissing and R. H. Müller. Solid lipid nanoparticles as carriers for sunscreens: in vitro release and in vivo skin penetration. J. Control Release 81:225-233 (2002).

50. U. Jacobi, H. Weigmann, M. Baumann, A. Reiche, W. Sterry, and J. Lademann. Lateral spreading of topically applied UV filter substances investigated by tape stripping. Skin Pharmacol. Appl. Skin Physiol. 17:17-22 (2004).

51. V. Sarveiya, S. Risk, and H. A. E. Benson. Liquid chromatographic assay for common sunscreen agents : application to in vivo assessment of skin penetration and systemic absorption in human volunteers. J. Chrom. B 803:225-231 (2004).

52. H. Lboutounne, J. F. Chaulet, C. Ploton, F. Falson, and F. Pirot. Sustained ex vivo skin antiseptic activity of chlorhexidine in poly ( $\varepsilon$-caprolactone) nanocapsule encapsulated form and as a digluconate. J. Control Release 82:319-334 (2002).

53. A. Rougier, D. Dupuis, C. Lotte, R. Roguet, and H. Schaefer. In vivo correlation between stratum corneum reservoir function and percutaneous absorption. J. Invest. Dermatol. 81:275278 (1983)

54. A. Rougier, C. Lotte, and H. I. Maibach. The measurement of the stratum corneum reservoir. A predictive method for in vivo percutaneous absorption studies: influence of application time. J. Invest. Dermatol. 83:460-462 (1984).

55. A. Rougier, C. Lotte, and D. Dupuis. An original predictive method for in vivo percutaneous absorption studies. J. Soc. Cos. Chem. 38:397-417 (1987).

56. A. Rougier, C. Lotte, and H. I. Maibach. In vivo percutaneous penetration of some organic compounds related to anatomic site in humans: predictive assessment by the stripping method. J. Pharm. Sci. 76:451-454 (1987).

57. D. Caron, C. Queille-Roussel, V. P. Shah, and H. Schaefer. Correlation between the drug penetration and the blanching effect of topically applied hydrocortisone creams in human beings. J. Am. Acad. Dermatol. 23:458-462 (1990). 
58. N. V. Sheth, M. B. McKeough, and S. L. Spruance. Measurements of the stratum corneum drug reservoir. J. Invest. Dermatol. 89:598-602 (1987).

59. C. Curdy, Y. N. Kalia, A. Naik, and R. H. Guy. Piroxicam delivery into human stratum corneum in vivo: iontophoresis versus passive diffusion. J. Control Release 76:73-79 (2001).

60. N. Keiko and Y. Toshiko. Volatilization of menthol, camphor and methyl salicylate from analgesic anti-inflammatory cataplasms and plasters. Yakuzaigaku. 47:168-175 (1987).

61. N. Goetz, G. Kaba, D. Good, G. Hussler, and P. Bore. Detection and identification of volatile compounds evolved from human hair and scalp using headspace gas chromatography. J. Soc. Cosmet. Chem. 39:1-13 (1988).

62. F. Pragst, V. Auwarter, B. Kiessling, and C. Dyes. Wipe-test and patch-test for alcohol misuse based on the concentration ratio of fatty acid ethyl esters and squalene CFAEE/CSQ in skin surface lipids. Forensic. Sci. Int. 143:77-86 (2004).

63. D. A. Bucks, J. J. Hostynek, R. S. Hinz, and R. H. Guy. Uptake of two zwitterionic surfactants into human skin in vivo. Toxicol. Appl. Pharmacol. 120:224-227 (1993).

64. F. Pirot, Y. N. Kalia, A. L. Stinchcomb, G. Keating, A. Bunge, and R. H. Guy. Characterization of the permeability barrier of human skin in vivo. Proc. Natl. Acad. Sci. U. S. A. 94:1562-1567 (1997).

65. M. B. Reddy, A. L. Stinchcomb, R. H. Guy, and A. L. Bunge. Determining dermal absorption parameters in vivo from tape strip data. Pharm. Res. 19:292-298 (2002).

66. H. A. Ayala-Bravo, D. Quintanar-Guerrero, A. Naik, Y. N. Kalia, J. M. Cornejo-Bravo, and A. Ganem-Quintanar. Effects of sucrose oleate and sucrose laureate on in vivo human stratum corneum permeability. Pharm. Res. 20:1267$1273(2003)$

67. H. C. Tsai, C. Y. Lin, H. M. Sheu, Y. L. Lo, and Y. H. Huang. Noninvasive characterization of regional variation in drug transport into human stratum corneum in vivo. Pharm. Res. 20:632-638 (2003).

68. V. P. Shah, G. L. Flynn, R. H. Guy, H. I. Maibach, H. Schaefer, J. P. Skelly, R. C. Wester, and A. Yacobi. In vivo percutaneous penetration/absorption. Pharm. Res. 8:1071-1075 (1991).

69. Y. C. Huang, L. Lesko, P. Schwartz, V. P. Shah, and R. Williams. Topical and transdermal generic drug products: regulatory issues and resolution. In K. R. Brain, K. A. Walters, and V. J. James (eds.), Prediction of Percutaneous Penetration, vol 3B, STS, Cardiff, 1993, pp. 463-472.

70. S. Shah, D. Hare, S. V. Dighe, and R. L. Williams. Bioequivalence of topical dermatological products. In V. P. Shah and H. I. Maibach (eds.), Topical Drug Bioavailability, Bioequivalence and Penetration, Plenum, New York, 1993, pp. 393-413.

71. V. P. Shah. Challenges in evaluating bioequivalence of dermatological drug products. In C. Surber, P. Elsner, and A. J. Birhcer (eds.), Exogenous Dermatology. Curr Prob Dermatol, Karger, Basel, 1995, pp. 152-157.

72. L. K. Pershing, J. L. Nelson, J. L. Corlett, S. P. Shrivastava, D. B. Hare, and V. P. Shah. Assessment of dermatopharmacokinetic approach in the bioequivalence determination of topical gel products. J. Am. Acad. Dermatol. 48:740-751 (2003).

73. D. P. Conner. Differences in DPK Methods. Available at: http:// www.fda.gov/ohrms/dockets/ac/01/slides/3804s2_05_conner/ index.htm, Advisory Committee for Pharmaceutical Sciences Meeting, Center for Drug Evaluation and Research (CDER), Food and Drug Administration (FDA), Rockville, MD, November 29 (2001)

74. T. J. Franz. Study \#1, Avita Gel 0.025\% vs Retin-A Gel 0.025\%, Advisory Committee for Pharmaceutical Sciences Meeting, Center for Drug Evaluation and Research (CDER), Food and Drug Administration (FDA), Rockville, MD, 2001 (November 29).

75. L. K. Pershing. Bioequivalence Assessment of three $0.025 \%$ tretinoin gel products: Dermatopharmacokinetic vs. Clinical Trial Methods, Advisory Committee for Pharmaceutical Sciences Meeting, Center for Drug Evaluation and Research (CDER), Food and Drug Administration (FDA), Rockville, MD, 2001 (November 29).
76. E. Chatelain, B. Gabard, and C. Surber. Skin penetration and sun protection factor of five UV filters: effect of the vehicle. Skin Pharmacol. Appl. Skin Physiol. 16:28-35 (2003).

77. R. Marks and R. P. Dawber. Skin surface biopsy: an improved technique for the examination of the horny layer. Br. J. Derm. 84:117-123 (1971).

78. J. M. Lachapelle, J. C. Gouverneur, M. Boulet, and D. Tennstedt. A modified technique (using polyester tape) of skin surface biopsy. Its interest for the investigation of athlete's foot. Br. J. Dermatol. 97:49-52 (1977).

79. Y. N. Kalia, F. Pirot, and R. H. Guy. Homogeneous transport in a heterogeneous membrane: water diffusion across human stratum corneum in vivo. Biophys. J 71:2692-2700 (1996).

80. Y. N. Kalia, I. Alberti, N. Sekkat, C. Curdy, A. Naik, and R. H. Guy. Normalization of stratum corneum barrier function and transepidermal water loss in vivo. Pharm. Res. 17:1148-1150 (2000).

81. E. Marttin, M. T. A. Neelissen-Subnel, F. H. N. HaanDe, and H. E. Boddé. A critical comparison of methods to quantify stratum corneum removed by tape stripping. Skin Pharmacol. 9:69-77 (1996).

82. F. Dreher, A. Arens, J. J. Hostynek, S. Mudumba, J. Ademola, and H. I. Maibach. Colorimetric method for quantifying Human stratum corneum removed by adhesive tape stripping. Acta Derm. Venereol. 78:186-189 (1998).

83. H. J. Weigmann, J. Lademann, H. Meffert, H. Schaefer, and W. Sterry. Determination of the horny layer profile by tape stripping in combination with optical spectroscopy in the visible range as a prerequisite to quantify percutaneous absorption. Skin Pharmacol. Appl. Skin Physiol. 12:34-45 (1999).

84. U. Lindemann, H. J. Weigmann, H. Schaefer, W. Sterry, and J. Lademann. Evaluation of the pseudo-absorption method to quantify human stratum corneum removed by tape stripping using protein absorption. Skin Pharmacol. Appl. Skin Physiol. 16:228-236 (2003).

85. H. J. Weigmann, U. Lindemann, C. Antoniou, G. N. Tsikrikas, A. I. Stratigos, A. Katsambas, W. Sterry, and J. Lademann. UV/VIS absorbance allows rapid, accurate and reproductible mass determination of corneocytes removed by tape stripping. Skin Pharmacol. Appl. Skin Physiol. 16:217-227 (2003).

86. Y. Chao, F. Nylander, and A. Leena. Determination of keratin protein in a tape-stripped skin sample from jet fuel exposed skin. Ann. Occup. Hyg. 48:65-73 (2004).

87. N. A. Puttnam. Attenuated total reflectance studies of the skin. J. Soc. Cosmet Chem. 23:209-226 (1972).

88. H. M. Klimisch and G. Chandra. Use of Fourier transform infrared spectroscopy with attenuated total reflectance for in vivo quantification of PDMS on human skin. J. Soc. Cosmet. Chem. 37:73-87 (1986).

89. V. H. W. Mak, R. O. Potts, and R. H. Guy. Percutaneous penetration enhancement in vivo measured by attenuated total reflectance infrared spectroscopy. Pharm. Res. 7:835-841 (1990).

90. D. Bommannan, R. Potts, and R. H. Guy. Examination of the effect of ethanol on human stratum corneum in vivo using infrared spectroscopy. J. Control Release 16:299-304 (1991).

91. N. Higo, A. Naik, D. B. Bommannan, R. O. Potts, and R. H. Guy. Validation of reflectance of reflectance infrared spectroscopy as a quantitative method to measure percutaneous absorption in vivo. Pharm. Res. 10:1500-1506 (1993).

92. D. Bommannan, R. Potts, and R. H. Guy. Examination of stratum corneum barrier function in vivo by infrared spectroscopy. J. Invest. Dermatol. 95:403-408 (1990).

93. M. Dias, J. Hadgraft, S. L. Raghavan, and J. Tetteh. The effect of solvent on permeant diffusion through membranes studied using ATR-FTIR and chemometric data analysis. J. Pharm. Sci. 93:186-196 (2004).

94. C. Herkenne, A. Naik, Y. N. Kalia, J. Hadgraft, and R. H. Guy. Dermatopharmacokinetic prediction of topical drug bioavailability in vivo. J. Invest. Dermatol. 127:887-894 (2007).

95. M. S. Roberts, Y. G. Anissimov, and R. A. Gonsalvez. Mathematical models in percutaneous absorption, In R. L. Bronaugh and H. I. Maibach (eds.), Percutaneous Absorption. Drugs, Cosmetics, Mechanisms, Methodology, Dekker, New York, 1999, pp. 3-55. 
96. J. Lademann, N. Otberg, H. Richter, U. Jacobi, H. Schaefer, U. Blume-Peytavi, and W. Sterry. Follicular penetration. An important pathway for topically applied substances. Der Hautarzt 54:321-323 (2003).

97. J. Lademann, H. Schaefer, N. Otberg, A. Teichmann, U. Blume-Peytavi, and W. Sterry. Penetration of microparticles into human skin. Der Hautarzt 55:1117-1119 (2004).

98. R. Toll, U. Jacobi, H. Richter, J. Lademann, H. Schaefer, and U. Blume-Peytavi. Penetration profile of microspheres in follicular targeting of terminal hair follicles. J. Invest. Dermatol. 123:168-176 (2004).

99. A. Teichmann, U. Jacobi, M. Ossadnik, H. Richter, S. Koch, W. Sterry, and J. Lademann. Differential stripping: determination of the amount of topically applied substances penetrated into hair follicles. J. Invest. Dermatol. 125:264-269 (2005).

100. M. Kreilgraad. Assessment of cutaneous drug delivery using microdialysis. Adv. Drug Deliv. Rev. 54:99-121 (2002).

101. E. Schnetz and M. Fartasch. Microdialysis for the evaluation of penetration through the human skin barrier-a promising tool for future research?. Eur. J. Pharm. Sci. 12:165-174 (2001).

102. P. Lonnroth, P. A. Jansson, and U. Smith. A microdialysis method allowing characterization of intercellular water space in humans. Am. J. Physiol. 253:E228-E231 (1987).

103. J. M. Ault, C. M. Riley, N. M. Meltzer, and C. E. Lunte. Dermal microdialysis sampling in vivo. Pharm. Res. 11:16311639 (1994).

104. F. X. Mathy, R. K. Verbeeck, and V. Préat. Validation of subcutaneous microdialysis sampling for pharmacokinetic studies on flurbiprofen in the rat. J. Pharm. Sci. 90:1897-1906 (2001).

105. L. Stahle. On mathematical models of microdialysis: geometry, steady-state models, recovery and probe radius. Adv. Drug Deliv. Rev. 45:149-167 (2000).

106. G. Fettweis and J. Borlak. Topics in xenobiochemistryapplication of microdialysis techniques in pharmacokinetic studies. Xenobiotica 26:473-485 (1996).

107. M. Kreilgaard. Dermal pharmacokinetics of microemulsion formulations determined by in vivo microdialysis. Pharm. Res. 18:367-373 (2001)

108. S. E. Cross, C. Anderson, M. J. Thompson, and M. S. Roberts. Is there tissue penetration after application of topical salicylate formulations?. Lancet 350:636, 1997 (1997).

109. S. E. Cross, C. Anderson, and M. S. Roberts. Topical penetration of commercial salicylate esters and salts using human isolated skin and clinical microdialysis studies. Br. J. Clin. Pharmacol. 46:29-35 (1998).

110. E. Benfeldt, J. Serup, and T. Menné. Effect of barrier perturbation on cutaneous salicylic acid penetration in human skin: in vivo pharmacokinetics using microdialysis and noninvasive quantification of barrier function. Br.J. Dermatol. 140:739-748 (1999).

111. F-X. Mathy, C. Lombry, R. K. Verbeeck, and V. Préat. Study of the percutaneous penetration of flurbiprofen by cutaneous and subcutaneous microdialysis after iontophoretic delivery in rat. J. Pharm. Sci. 94:144-152 (2005).

112. F.-X. Mathy, D. Ntivunwa, R. K. Verbeeck, and V. Préat. Fluconazole distribution in rat dermis following intravenous and topical application: a microdialysis study. J. Pharm. Sci. 94:770-780 (2005).

113. D. McCleverty, R. Lyons, and B. Henry. Microdialysis sampling and the clinical determination of topical dermal bioequivalence. Int. J. Pharm. 308:1-7 (2006).

114. K. Matsuyama, M. Nakashima, M. Ichikawa, T. Yano, S. Satoh, and S. Goto. In vivo microdialysis for the transdermal absorption of valproate in rats. Biol. Pharm. Bull. 17:13951398 (1994).

115. K. Matsuyama, M. Nakashima, Y. Nakaboh, M. Ichikawa, T. Yano, and S. Satoh. Application of in vivo microdialysis to transdermal absorption of methotrexate in rats. Pharm. Res. 11:684-686 (1994).

116. M. Nakashima, M. F. Zhao, H. Ohya, M. Sakurai, H. Sasaki, K. Matsuyama, and M. Ichikawa. Evaluation of in vivo transdermal absorption of cyclosporin with absorption enhancer using intradermal microdialysis in rats. J. Pharm. Pharmacol. 48:1143-1146 (1996).

117. T. Murakami, M. Yoshioka, R. Yumoto, Y. Higashi, S. Shigeki, Y. Ikuta, and N. Yata. Topical delivery of keloid therapeutic drug, tranilast, by combined use of oleic acid and propylene glycol as a penetration enhancer: evaluation by skin microdialysis in rats. J. Pharm. Pharmacol. 50:49-54 (1998).

118. T. Murakami, M. Yoshioka, I. Okamoto, R. Yumoto, Y. Higashi, K. Okahara, and N. Yata. Effect of ointment bases on topical and transdermal delivery of salicylic acid in rats: evaluation by skin microdialysis. J. Pharm. Pharmacol. 50:5561 (1998)

119. J. Y. Fang, L. R. Hsu, Y. B. Huang, and Y. H. Tsai. Evaluation of transdermal iontophoresis of enoxacin from polymer formulations: in vitro skin permeation and in vivo microdialysis using Wistar rat as an animal model. Int. J. Pharm. 180:137-149 (1999).

120. J. Y. Fang, K. C. Sung, H. H. Lin, and C. L. Fang. Transdermal iontophoretic delivery of diclofenac sodium from various polymer formulations: in vitro and in vivo studies. Int. J. Pharm. 178:83-92 (1999).

121. J. Y. Fang, C. T. Kuo, C. L. Fang, Y. B. Huang, and Y. Tsai. Transdermal iontophoresis of sodium nonivamide acetate evaluated by in vivo microdialysis and histologic study. Drug Dev. Res. 46:87-95 (1999).

122. P. Ding, H. Xu, G. Wei, and J. Zheng. Microdialysis sampling coupled to HPLC for transdermal delivery study of ondansetron hydrochloride in rats. Biomed. Chromatogr. 14:141-143 (2000).

123. E. Benfeldt and J. Serup. Effect of barrier perturbation on cutaneous penetration of salicylic acid in hairless rats: in vivo pharmacokinetics using microdialysis and non-invasive quantification of barrier function. Arch. Dermatol. Res. 291:517-526 (1991).

124. C. Anderson, T. Andersson, and M. Molander. Ethanol absorption across human skin measured by in vivo microdialysis technique. Acta Derm. Venerol (Stockh) 71:389-393 (1991).

125. W. F. Elmquist and R. J. Sawchuk. Application of microdialysis in pharmacokinetic studies. Pharm. Res. 14:267-288 (1997).

126. D. K. Hansen, M. I. Davies, S. Lunte, and C. E. Lunte. Pharmacokinetic and metabolism studies using microdialysis sampling. J. Pharm. Sci. 88:14-27 (1999).

127. L. Hegemann, C. Forstinger, B. Partsch, I. Lagler, S. Krotz, and K. Wolff. Microdialysis in cutaneous pharmacology: Kinetic analysis of transdermally delivered nicotine. J. Invest. Dermatol. 104:839-843 (1995).

128. M. Müller, R. Schmid, O. Wagner, B. Osten, H. Shayganfar, and $\mathrm{H}$. G. Eichler. In vivo characterization of transdermal drug transport by microdialysis. J. Control Release 37:49-57 (1995).

129. E. Benfeldt and L. Groth. Feasibility of measuring lipophilic or protein-bound drugs in the dermis by in vivo microdialysis after topical or systemic drug administration. Acta Derm. Venereol. 78:274-278 (1998).

130. M. Müller, H. Mascher, C. Kikuta, S. Schäfer, M. Brunner, G. Dorner, and H. G. Eichler. Diclofenac concentrations in defined tissue layers after topical administration. Clin. Pharmacol. Ther. 62:293-299 (1997)

131. M. Müller, M. Brunner, R. Schmid, E. M. Putz, A Schmiedberger, I. Wallner, and G. Eichler. Comparison of three different experimental methods for the assessment of peripheral compartment pharmacokinetic in humans. Life Sci. 62:227-234 (1998).

132. I. Tegeder, U. Muth-Selbach, J. Lötsch, G. Rüsing, R. Oelkers, K. Brune, S. Meller, G. R. Kelm, F. Sörgel, and G. Geisslinger. Application of microdialysis for the determination of muscle and subcutaneous tissue concentrations after oral and topical ibuprofen administration. Clin. Pharmacol. Ther. 65:357-368 (1999)

133. G. Stagni, D. O’Donnel, Y. J. Liu, D. L. Kellog Jr, T. Morgan, and A. M. M. Shepherd. Intradermal microdialysis: kinetics of 
iontophoretically delivered propanolol in forearm dermis. $J$. Control Release 63:331-339 (2000).

134. G. Stagni, M. Ehsan Ali, and D. Weng. Pharmacokinetics of acyclovir in rabbit skin after i.v.-bolus, ointment, and iontophoretic administrations. Int. J. Pharm. 274:201-211 (2004).

135. C. J. Morgan, A. G. Renwick, and P. S. Friedmann. The role of stratum corneum and dermal microvascular perfusion in penetration and tissue levels of water-soluble drugs investigated by microdialysis. Br. J. Derm. 148:434-443 (2003).

136. L. Groth, A. Jorgensen, and J. Serup. Cutaneous microdialysis in the rat: insertion trauma and effect of anaesthesia studied by laser Doppler perfusion imaging and histamine release. Skin Pharmacol. Appl. Skin Physiol. 11:12-132 (1998).

137. L. Groth and J. Serup. Cutaneous microdialysis in man: effects of needle insertion trauma and anaesthesia on skin perfusion, erythema and skin thickness. Acta Derm. Venereol. 78:5-9 (1998).

138. F.-X. Mathy, A.-R. Denet, B. Vroman, P. Clarijs, A. Barel, R.K. Verbeeck, and V. Préat. In vivo tolerance assessment of skin after insertion of subcutaneous and cutaneous microdialysis probes in the rat. Skin Pharmacol. Appl. Skin Physiol. 16:18-27 (2003)

139. C. Anderson, T. Andersson, and K. Wardell. Changes in skin circulation after insertion of a microdialysis probe visualized by laser Doppler perfusion imaging. J. Invest. Dermatol. 102:807811 (1994).

140. L. J. Petersen, P. S. Skov, C. Bindslev-Jensen, and J. Sondergaard. Histamine release in immediate-type hypersensitivity reactions in intact human skin measured by microdialysis. A preliminary study. Allergy 47:635-637 (1992).

141. L. J. Petersen. Measurement of histamine release in intact human skin by microdialysis technique, clinical and experimental findings. Danish Med. Bull. 45:383-401 (1998).

142. J. A. Stenken. Methods and issues in microdialysis calibration. Anal. Chim. Acta. 379:337-358 (1999).

143. A. L. Krogstad, P. A. Jansson, P. Gisslen, and P. Lonnroth. Microdialysis methodology for the measurement of dermal interstitial fluid in humans. Br. J. Dermatol. 134:1005-1012 (1996).

144. S. Menacherry, W. Hubert, and J. B. Justice. In vivo calibration of microdialysis probes for exogenous compounds. Anal. Chem. 64:577-583 (1992).

145. L. Stahle, S. Segersvard, and U. Ungerstedt. A comparison between three methods for estimation of extracellular concentrations of exogenous and endogenous compounds by microdialysis. J. Pharmacol. Meth. 25:41-52 (1991).

146. C. I. Larsson. The use of an "internal standard" for control of the recovery in microdialysis. Life Sci. 49:PL73-PL78 (1991).

147. D. Scheller and J. Kolb. The internal reference technique in microdialysis: a practical approach to monitoring dialysis efficiency and to calculating tissue concentration from dialyzate samples. J. Neurosci. Methods 40:31-38 (1991).

148. C. Carneheim and L. Staahle. Microdialysis of lipophilic compounds: a methodological study. Pharmacol. Toxicol. 69:378-380 (1991).

149. Y. Kurosaki, S. Nakamura, Y. Shiojiri, and H. Kawasaki. Lipomicrodialysis: a new microdialysis method for studying the pharmacokinetics of lipophilic substances. Biol. Pharm. Bull. 21:194-196 (1998).

150. M. I. Davies, J. D. Cooper, S. S. Desmond, C. E. Lunte, and S. Lunte. Analytical considerations for microdialysis sampling. Adv. Drug Deliv. Rev. 45:169-188 (2000).
151. I. Tegeder, L. Brautigam, M. Podda, S. Meier, R. Kaufmann, G. Geisslinger, and M. Grundmann-Kollmann. Time course of 8-methoxypsoralen concentrations in skin and plasma after topical (bath and cream) and oral administration of 8-methoxypsoralen. Clin. Pharmacol. Ther. 71:153-161 (2002).

152. C. Schrolnberger, M. Brunner, B. X. Mayer, H. G. Eichler, and M. Müller. Application of the minimal trauma tissue biopsy to transdermal clinical pharmacokinetric studies. J. Control Release 75:297-306 (2001).

153. G. Volden, A. K. Thorsrud, I. Bjornson, and E. Jellum. Biochemical composition of suction blister fluid determined by high resolution multicomponent analysis (capillary gas chromatography-mass spectrometry and two-dimensional electrophoresis). J. Invest. Dermatol. 75:421-424 (1980).

154. P. Svedman and C. Svedman. Skin mini-erosion sampling technique: feasibility study with regard to serial glucose measurement. Pharm. Res. 15:883-888 (1998).

155. S. Makki, P. Treffel, P. Humbert, and P. Agache. High performance liquid chromatographic determination of citropten and bergapten in suction blister fluid after solar product application in humans. J. Chromatogr. 563:407-413 (1991).

156. P. Treffel, S. Makki, B. Faivre, P. Humbert, D. Blanc, and P. Agache. Citropten and bergapten suction blister fluid concentrations after solar product application in man. Skin Pharmacol. 4:100-108 (1991).

157. C. Surber, K. P. Wilhelm, D. Bermann, and H. I. Maibach. In vivo skin penetration of acitretin in volunteers using three sampling techniques. Pharm. Res. 10:1291-1294 (1993).

158. R. J. Scheuplein. Mechanism of percutaneous absorption II: Transient diffusion and the relative importance of various routes of skin penetration. J. Invest. Dermatol. 48:79-88 (1967).

159. A. Rolland, N. Wagner, A. Chatelus, B. Shroot, and H. Schaefer. Site-specific drug delivery to pilosebaceous structures using polymeric microspheres. Pharm. Res. 10:1738-1744 (1993).

160. R. A. Bojar, A. G. Cutclife, L. Graupe, W. J. Cunliffe, and K. T. Holland. Follicular concentrations of azelaic acid after a single topical application. Br. J. Dermatol. 129:399-402 (1993).

161. O. H. Mills Jr and A. M. Kligman. A human model for assaying comedolytic substances. Br. J. Derm. 107:543-548 (1982).

162. N. Otberg, H. Richter, A. Knuttel, H. Schaefer, W. Sterry, and J. Lademann. Laser spectroscopic methods for the characterization of open and closed follicles. Laser Phys. Lett. 1:46-49 (2004).

163. P. J. Caspers, G. W. Lucassen, E. A. Carter, H. A. Bruining, and G. J. Puppels. In vivo confocal Raman microspectroscopy of the skin: noninvasive determination of molecular concentration profiles. J. Invest. Dermatol. 116:434-442 (2001).

164. L. Chrit, P. Bastien, B. Biatry, J. T. Simonnet, A. Potter, A. M. Minondo, F. Flament, R. Bazin, G. D. Sockalingum, F. Leroy, $\mathrm{M}$. Manfait, and C. Hadjur. In vitro and in vivo confocal Raman study of human skin hydration: assessment of a new moisturizing agent, pMPC. Biopolymers. 85:359-369 (2007).

165. L. Chrit, P. Bastien, G. D. Sockalingum, D. Batisse, F. Leroy, M. Manfait, and C. Hadjur. An in vivo randomized study of human skin moisturization by a new confocal Raman fiberoptic microprobe: assessment of a glycerol-based hydration cream. Skin Pharmacol. Physiol. 19:207-215 (2006).

166. V. Wascotte, P. Caspers, J. de Sterke, M. Jadoul, R. H. Guy, and V. Préat. Assessment of the "skin reservoir" of urea by confocal Raman microspectroscopy and reverse iontophoresis in vivo. Pharm. Res, in press (2007). 